\title{
Taxonomy of asteroid families among the Jupiter Trojans: comparison between spectroscopic data and the Sloan Digital Sky Survey colors
}

\author{
F. Roig ${ }^{1}$, A. O. Ribeiro ${ }^{1}$, and R. Gil-Hutton ${ }^{2}$ \\ 1 Observatório Nacional, Rua General José Cristino 77, Rio de Janeiro, 20921-400, Brazil \\ e-mail: froig@on.br \\ ${ }^{2}$ Complejo Astronómico El Leoncito (CASLEO) and Universidad Nacional de San Juan, Av. España 1512 sur, San Juan, J5402DSP, \\ Argentina
}

Received 1 December 2007 / Accepted 22 February 2008

\begin{abstract}
Aims. We present a comparative analysis of the spectral slope and color distributions of Jupiter Trojans, with particular attention to asteroid families. We use a sample of data from the Moving Object Catalog of the Sloan Digital Sky Survey, together with spectra obtained from several surveys.

Methods. We extracted a first sample of 349 observations, corresponding to 250 Trojan asteroids, from the Sloan Digital Sky Survey, and a second sample of 138 spectra, corresponding to 115 Trojans, from the literature. We computed the spectral slopes in the first sample by means of a least-squares fit to a straight line of the fluxes obtained from the Sloan observations, and in the second sample by means of a fit to the rebinned spectra. In both cases the reflectance fluxes/spectra were renormalized to 1 at $6230 \AA$.

Results. We found that the distribution of spectral slopes among Trojan asteroids shows a bimodality. About $2 / 3$ of the objects have reddish slopes compatible with D-type asteroids, while the remaining bodies show less reddish colors compatible with the P-type and C-type classifications. The members of asteroid families also show a bimodal distribution with a very slight predominance of D-type asteroids, but the background is clearly dominated by the D-types. The L4 and L5 swarms show different distributions of spectral slopes, and bimodality is only observed in L4. These differences can be attributed to the asteroid families since the background asteroids show the same slope distributions in both swarms. The analysis of individual families indicates that the families in L5 are taxonomically homogeneous, but in L4 they show a mixture of taxonomic types. We discuss a few scenarios that might help to interpret these results.
\end{abstract}

Key words. minor planets, asteroids

\section{Introduction}

Trojan asteroids are an interesting population of minor bodies due to their dynamical characteristics and physical properties. The main hypotheses about the origin of the Jupiter Trojans assumed that they formed either during the final stages of the planetary formation (Marzari \& Scholl 1998), or during the epoch of planetary migration (Morbidelli et al. 2005), in any case more than 3.8 Gy ago. The dynamical configuration kept the Trojans isolated from the asteroid Main Belt throughout the history of the Solar System. In spite of eventual interactions with other populations of minor bodies like the Hildas, the Jupiter family comets, and the Centaurs, their collisional evolution has been dictated mostly by the intrapopulation collisions (Marzari et al. 1996, 1997). Therefore, the Jupiter Trojans may be considered primordial bodies, whose dynamical and physical properties can provide important clues about the environment of planetary formation.

Several studies have addressed the dynamical properties of the Trojan population. Of particular interest for the present work are the papers by Milani (1993) and Beaugé \& Roig (2001), who computed proper elements for a large number of Jupiter Trojans and realized the existence of several dynamical families. These authors found that the families are mostly concentrated at the L4 swarm, and they are much less conspicuous in the L5 swarm.

On the other hand, spectrophotometry has been used by different authors to provide information about the surface physical properties of the Jupiter Trojans. Zellner et al. (1985) provided the first multiband photometric observations of 21 of these objects. This allowed them to be classified within the $\mathrm{D}$ and $\mathrm{P}$ taxonomic classes (Tholen 1989), with a significant predominance of the D class (about $90 \%$ of bodies). Jewitt \& Luu (1990) obtained spectra in the visible range of 32 Trojans and concluded that they show significant analogies with the spectra of cometary nuclei. Eighteen Trojan asteroids have been observed in the framework of the two major spectroscopic surveys: the SMASS (Small Mainbelt Asteroids Spectroscopic Survey, Xu et al. 1995; Bus \& Binzel 2002) and the S3OS2 (Small Solar System Objects Spectroscopic Survey, Lazzaro et al. 2004). Analyzing data from this latter survey, Carvano et al. (2003) concluded that Trojan spectra show differences in spectral slope with respect to the population of D type asteroids in the main belt. Specific surveys have also helped to increase the spectral data of Jupiter Trojans in the visible. Bendjoya et al. (2004) contributed with observations of 34 Trojans; Fornasier et al. (2004) observed 24 members of asteroid families detected in the L5 swarm; Dotto et al. (2006) reported observations of 13 members of asteroid families in the 
L4 swarm; and finally, Fornasier et al. (2007) provided spectra of other 47 members of families in both swarms. Spectra in the near infrared (NIR) have been obtained by Luu et al. (1994), and more recently, by Dotto et al. (2006) who observed 24 Trojans belonging to the main asteroid families in L4 and L5. Surface mineralogy based on NIR spectra of 20 Trojans has been analyzed by Emery \& Brown (2003), and recently by Yang \& Jewitt (2007) who addressed the presence of water ice on the Trojan surfaces. A quite complete analysis of the properties of Jupiter Trojans observed by the Sloan Digital Sky Survey (SDSS) has been developed by Szabó et al. (2007), who addressed an interesting correlation between colors and orbital inclinations. All these studies indicate that Jupiter Trojans seems to be a quite homogeneous population in terms of taxonomy and surface mineralogy.

In spite of these works, the amount of spectroscopic data of Jupiter Trojans presently available is still too small to allow a statistical analysis of the taxonomic properties of these bodies. Moreover, since spectra come from different sources they do not constitute a homogeneous data sample. In this paper, we analyze the taxonomy of Jupiter Trojans with data contained in the 3rd release of the SDSS Moving Objects Catalog (MOC3), and compare the results to the available spectroscopic data, with particular emphasis on Trojan asteroid families. We recall that an asteroid family is the outcome of the catastrophic breakup of an asteroid. Therefore, the family members carry information about the mineralogical composition of the corresponding parent body, and the study of these members may help to better understand the formation and evolution not only of the parent body but also of the whole Trojan population. The SDSS-MOC3 colors have proved to be a useful tool to characterize the taxonomy of Main Belt asteroids, as recently addressed by Roig \& Gil-Hutton (2006); Binzel et al. (2006, 2007); Duffard \& Roig (2007); Roig et al. (2008); and Gil-Hutton \& Brunini (2008).

The paper is organized as follows: Sect. 2 introduces the two data samples used in this study and compares their internal accuracy. Section 3 is devoted to the global analysis of the color and taxonomy distributions of the data samples. Section 4 concentrates on the particular analysis of selected asteroid families. Finally, Sect. 5 contains the conclusions.

\section{Selection of the data samples}

In this work, we will analyze two different data sets containing information on Trojan asteroids taxonomy. They are described in the following.

\subsection{The Sloan sample}

The first data set is constituted by observations from the SDSSMOC3 and their selection required some care. The SDSS-MOC3 includes photometric measurements of more than 204000 moving objects, of which only 67637 observations have been effectively linked to 43424 unique known asteroids. The observations consist of calibrated magnitudes in the $u, g, r, i, z$ system of filters, centered at 3540, 4770, 6230, 7630, and $9130 \AA$, respectively, and with bandwidths of $\sim 100 \AA$ (Fukugita et al. 1996). We adopted here a procedure similar to that of Roig \& GilHutton (2006). First, we used the solar colors provided by Ivezić et al. (2001) to compute the reflectance fluxes $F$ in the five bands, normalized to 1 at the $r$ band. Then, we discarded the observations with error $>10 \%$ in any of the $F_{g}, F_{r}, F_{i}$, and $F_{z}$ fluxes. Observations showing anomalous values of the fluxes, like $F_{u}>1.0, F_{g}>1.3, F_{i}>1.5, F_{z}>1.7$, and $F_{g}<0.6$

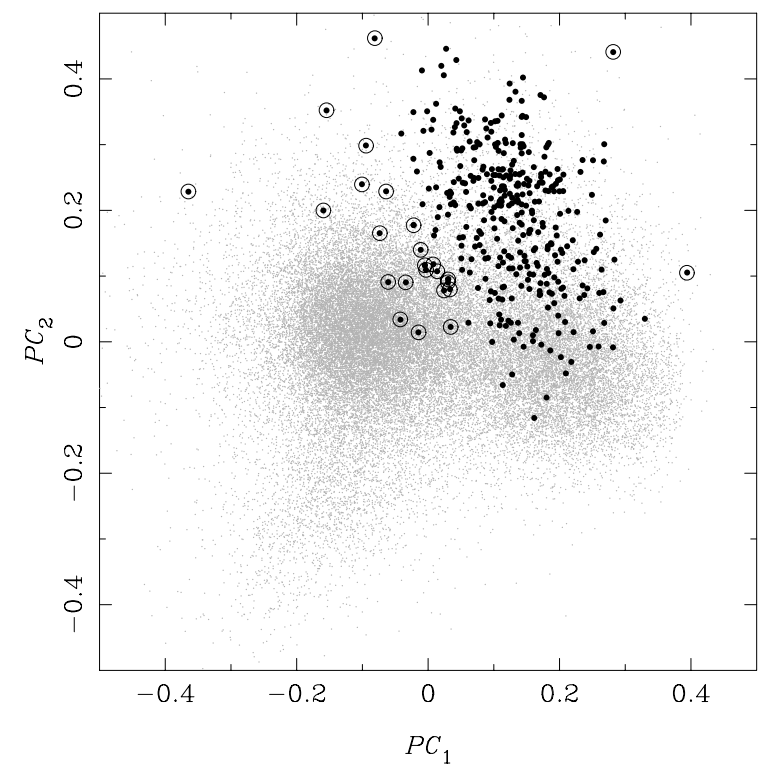

Fig. 1. Distribution of 40863 observations selected from the SDSSMOC3 (gray dots) in the space of first and second principal components. The black dots correspond to 371 observations of known Jupiter Trojans, but those surrounded by a circle have been discarded (see text).

were also discarded. Note that the error in $F_{u}$ has not been constrained, which allows us to obtain a final data set with more than twice the amount of observations than if we had restricted this error to less than $10 \%$. As we will explain later in this section, this error is not critical for our study.

We ended up with a sample of 40863 observations, corresponding to 28910 unique known asteroids. The distribution of these observations in the space of principal components is shown in Fig. 1 (gray dots), where the first and second principal components, $P C_{1}$ and $P C_{2}$, have been computed from the reflectance fluxes as:

$P C_{1}=0.886 F_{u}+0.416 F_{g}-0.175 F_{i}+0.099 F_{z}-0.849$
$P C_{2}=-0.049 F_{u}-0.003 F_{g}+0.284 F_{i}+0.957 F_{z}-1.261$

The use of principal components allows for an easy interpretation of the observations in a bidimensional space. Observations with $P C_{1} \gtrsim 0$ correspond to featureless spectra (e.g., C-, X-, and D-type asteroids), while those with $P C_{1} \lesssim 0$ correspond to featured spectra that show a broad absorption band longwards of $7000 \AA$ (e.g. S- and V-type asteroids). The value of $P C_{2}$ is related to the overall slope of the spectrum, the larger the $P C_{2}$, the higher the slope. For featureless spectra, $P C_{2}$ gives an idea of how reddish is the spectrum; for featured spectra, it gives an idea of the band depth (see Roig \& Gil-Hutton 2006).

Within these 40863 observations, we identified 371 observations corresponding to 257 different Trojan asteroids listed in the database of Trojan proper elements maintained by the PETrA Project (Beaugé \& Roig 2001; http: //staff.on.br/froig/ petra). Their distribution in the space of principal components is also shown in Fig. 1 (black dots). Most of these observations of Trojan asteroids have values of $P C_{1} \gtrsim 0$ compatible with featureless spectra, and values of $P C_{2} \gtrsim 0$, indicating that they have moderate to high spectral slopes. There are, however, some observations (circled dots in Fig. 1) that either depart significantly from the overall distribution of other Trojan observations, or clearly fall within the region of featured spectra occupied by the S-type asteroids $\left(P C_{1} \lesssim 0.05\right.$ and $\left.P C_{2} \lesssim 0.2\right)$. Direct inspection of the reflectance fluxes indicated that these observations are 
not compatible with featureless spectra or that they show anomalous fluctuations, therefore, we discarded them as well. The final sample contains 349 observations, corresponding to 250 unique known Trojan asteroids. Hereafter, we will refer to this sample as the Sloan sample. The Sloan sample includes 200 observations of asteroids in the L4 swarm and 149 observations of the L5 swarm. About $40 \%$ of these observations correspond to asteroid family members.

It is worth mentioning that the main goal of our selection method is that it provides a sample of good quality observations from the SDSS-MOC3 that can easily be linked to family and to background (i.e., nonfamily) asteroids. Our approach is different from that introduced by Szabó et al. (2007), who applied a kinematic criterion to select candidate Trojan asteroids within the SDSS-MOC3. These authors got a much larger sample of 1187 observations, but these observations cannot be separated in those corresponding to family and to nonfamily asteroids.

Each observation in our Sloan sample has been characterized by its equivalent spectral slope $S$, in $\AA^{-1}$. We computed this slope from a linear least-squares fit to a straight line passing through the fluxes $F_{g}, F_{r}, F_{i}$, and $F_{z}$. This fit took into account the individual errors $\Delta F$ of the fluxes to estimate the slope and its error, $\Delta S$. Hereafter, we will refer to this set of 349 slopes as the Sloan slopes. Note that the flux $F_{u}$ has not been used to compute the spectral slope. The reason for this is twofold: (i) we know, from spectroscopic observations, that the reflectance flux in the $u$ band usually drops off and significantly deviates from the linear trend of the spectrum; (ii) we intend to compare the Sloan sample to a sample of spectroscopic data, described below, where most spectra do not cover the wavelengths $\lesssim 5000 \AA$. Since $F_{u}$ does not contribute effectively to determine the slope, there is no harm in keeping its error unconstrained as we did.

Table 1 provides the list of all the known Trojan asteroids contained in our Sloan sample. This table also gives the estimated spectral slope, $S$, with its correspondig error, $\Delta S$, and the number of observations, $N_{\text {obs }}$, in the sample. For $N_{\text {obs }} \geq 2$, the slope given in this table is the weighted mean of the individual Sloan slopes, with the weights defined as $1 /(\Delta S)^{2}$.

\subsection{The spectroscopic sample}

The second data set analyzed here is a collection of 138 spectra corresponding to 115 individual Trojan asteroids published in the literature. All the spectra are defined in the visible wavelength range and have been obtained by different observational surveys, in particular: Three spectra come from the SMASS1 survey (Xu et al. 1995); 2 spectra from the SMASS2 survey (Bus \& Binzel 2002); 33 spectra from Bendjoya et al. (2004); 25 spectra from Fornasier et al. (2004); 13 spectra from the S3OS2 survey (Lazzaro et al. 2004); 15 spectra from Dotto et al. (2006); and 47 spectra from Fornasier et al. (2007). Hereafter, we will refer to this data set as the Spectroscopic sample. This sample includes 76 spectra of asteroids in the L4 swarm and 62 spectra of the L5 swarm. About $70 \%$ of these spectra correspond to asteroid family members.

To determine the spectral slope we proceeded as follows. First, we restricted all the spectra to the interval 5000-9200 A. This wavelength interval is similar to the one adopted to compute the Sloan slopes, and it is well covered by most spectra in our sample, except for a few cases for which we had to work with a smaller available range. Then, we divided this interval into 42 equal subintervals, or channels, of $100 \AA$ in width. At each channel, we computed the average reflectance flux $\bar{F}$, its standard deviation $\sigma_{\mathrm{F}}$ and the central wavelength $\lambda_{\mathrm{c}}$, and we attributed to $\bar{F}$ an observational error $\Delta F= \pm 3 \sigma_{\mathrm{F}}$. Then, we normalized the set of fluxes $\bar{F}\left(\lambda_{\mathrm{c}}\right)$ to 1 at $6230 \AA$, to make them comparable to the Sloan fluxes (this normalization was done preserving the relative error $\Delta F / \bar{F})$. Finally, we computed the slope of these normalized data from a linear least-squares fit to a straight line passing through the 42 channels. This fit took into account the individual observational errors $\Delta F$ to estimate the slope of the fit, $S$, and its error, $\Delta S$. Note that, with this procedure, we accounted for the noise of the spectrum to estimate $\Delta S$ because the more noisy the spectrum, the larger the values of $\Delta F$ and so the larger the slope error. We believe that this approach is more realistic than the one used by Fornasier et al. (2007), who guessed an "ad-hoc" error of $\pm 5 \times 10^{-6} \AA^{-1}$, aiming to account for uncertainties in the sample related to the use of data obtained by different surveys. We must recall that, in most cases, our estimated error $\Delta S$ is much larger than the error we would have estimated using the Fornasier et al. approach.

It is also worth noting that the slopes computed here are not compatible with other published slopes (e.g., Jewitt \& Luu 1990; Fornasier et al. 2007) due to different normalization wavelengths - usually $5500 \AA$ - and also due to different wavelengths intervals used to fit the data. In fact, our slopes may be up to $20 \%$ smaller than those published in the literature. Hereafter, we will refer to our set of 138 slopes as the Spectroscopic slopes, to distinguish them from the Sloan slopes.

Table 2 provides the list of all the known Trojan asteroids contained in our Spectroscopic sample. For asteroids with $N_{\text {obs }} \geq 2$, the slope shown in this table has been computed as the weighted mean of the individual Spectroscopic slopes, with the weights defined as $1 /(\Delta S)^{2}$. It is worth recalling that in Table 1 , the same survey, i.e. the SDSS, made all the observations of the asteroids with $N_{\text {obs }} \geq 2$, while in Table 2 different spectroscopic surveys made the observations of the asteroids with $N_{\text {obs }} \geq 2$ (except the ones indicated by an asterisk).

\subsection{Accuracy of the samples}

The Sloan sample is $\sim 2.5$ times larger than the Spectroscopic sample, which in terms of statistics does not appear to be a significant improvement. However, the Sloan sample is expected to be more homogeneous than the Spectroscopic sample because, in the former case, the observations come from the same survey, while in the latter they come from different surveys. Moreover, the spectroscopic surveys have usually been dedicated either to observe only family members (e.g., Fornasier et al. 2004; Dotto et al. 2006; Fornasier et al. 2007), or to observe only background asteroids (e.g., Lazzaro et al. 2004; Bendjoya et al. 2004). But the Sloan sample includes both family members and background asteroids observed by the same survey. We also expect that the Sloan sample includes a significant amount of very small Trojans that spectroscopic surveys normally do not observe. Although the SDSS photometry is not as precise as spectroscopy, this is not crucial in the case of the Trojan asteroids because they all show featureless spectra that are properly characterized by the average spectral slope.

In order to verify the reliability of the Sloan and the Spectroscopic samples, we performed the following test. For each asteroid with $N_{\text {obs }} \geq 2$ in Table 1, we computed the parameter

$$
\epsilon=\frac{\left|S_{1}-S_{2}\right|}{\Delta S_{1}+\Delta S_{2}}
$$




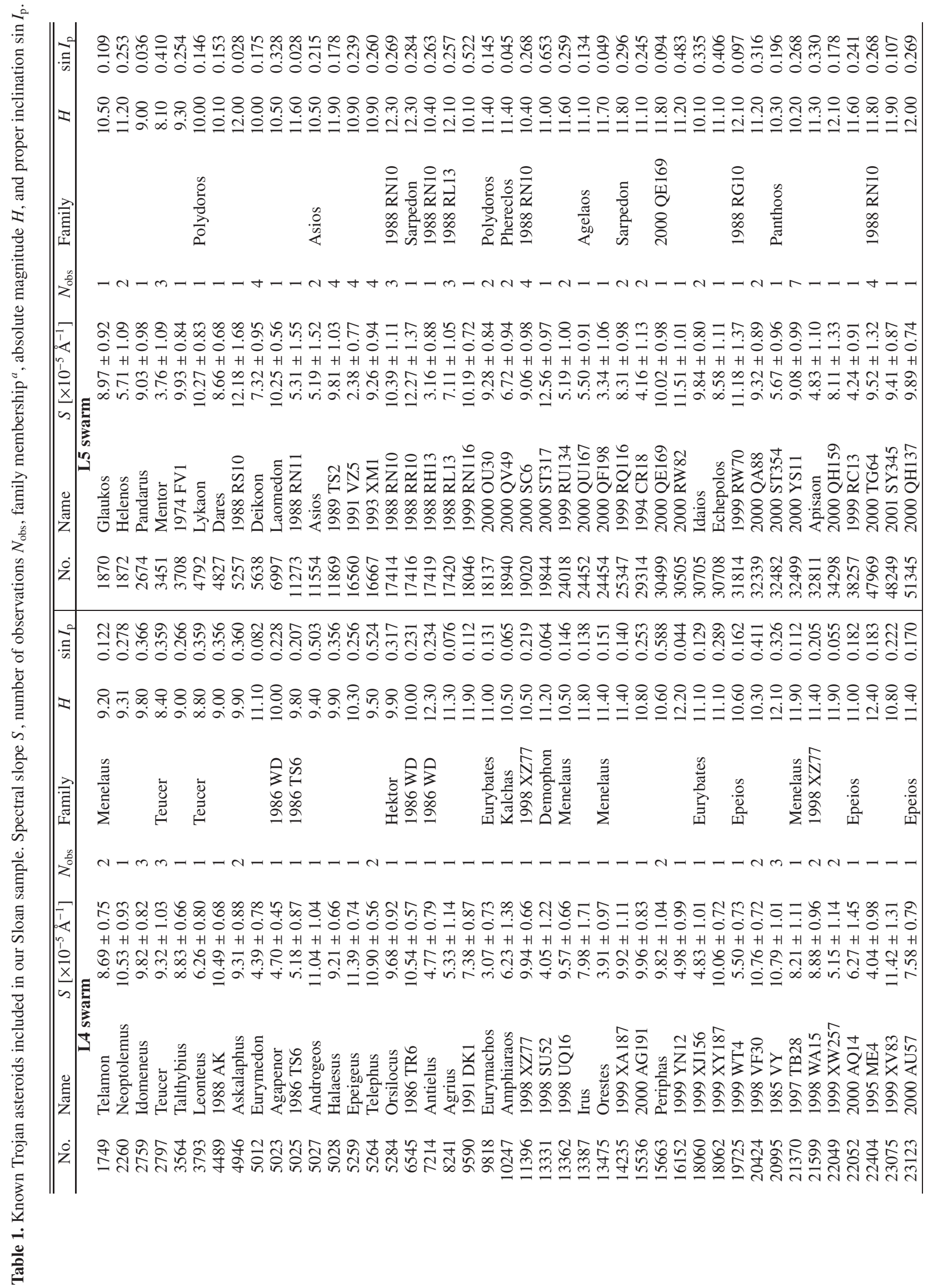




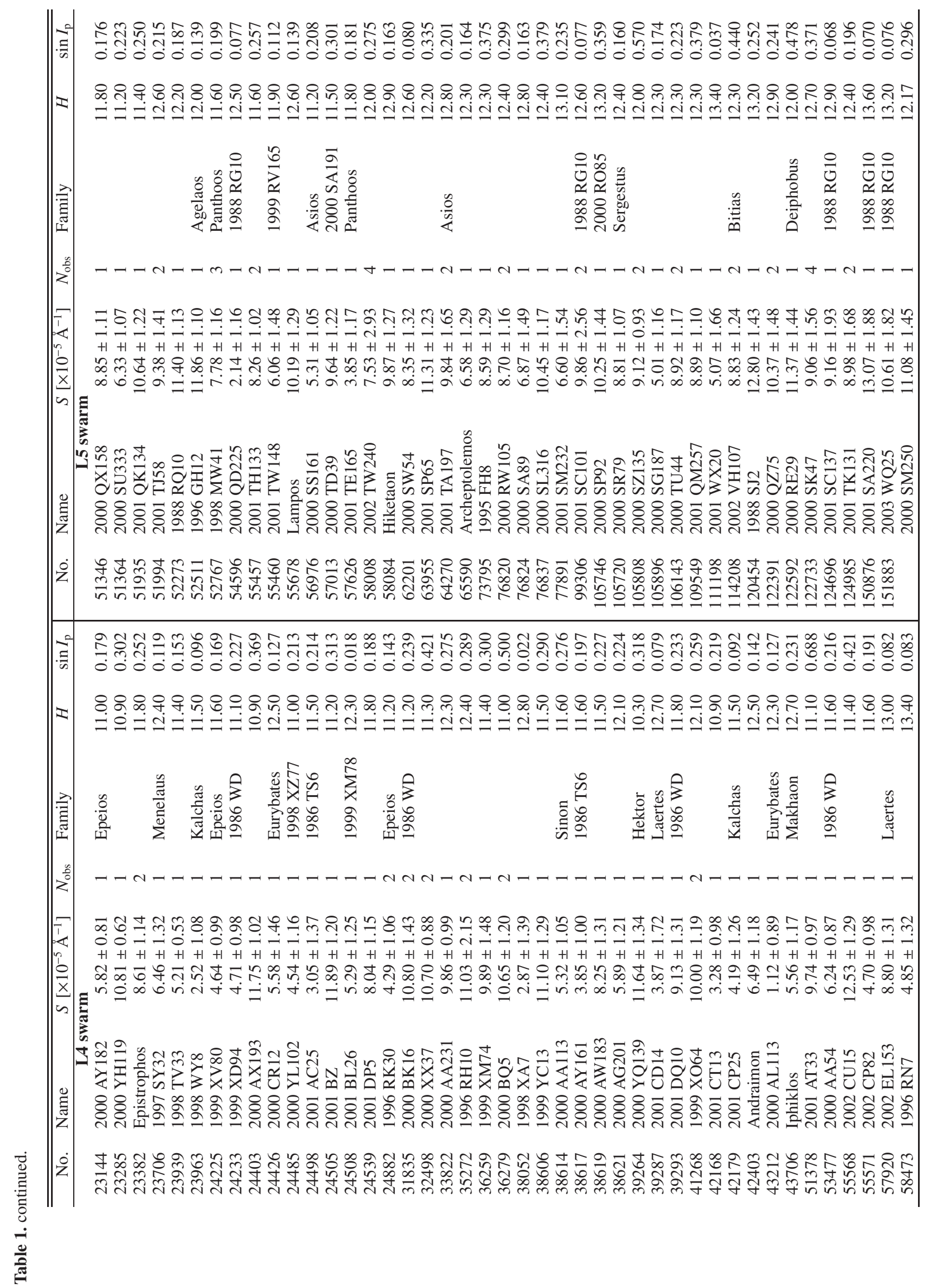




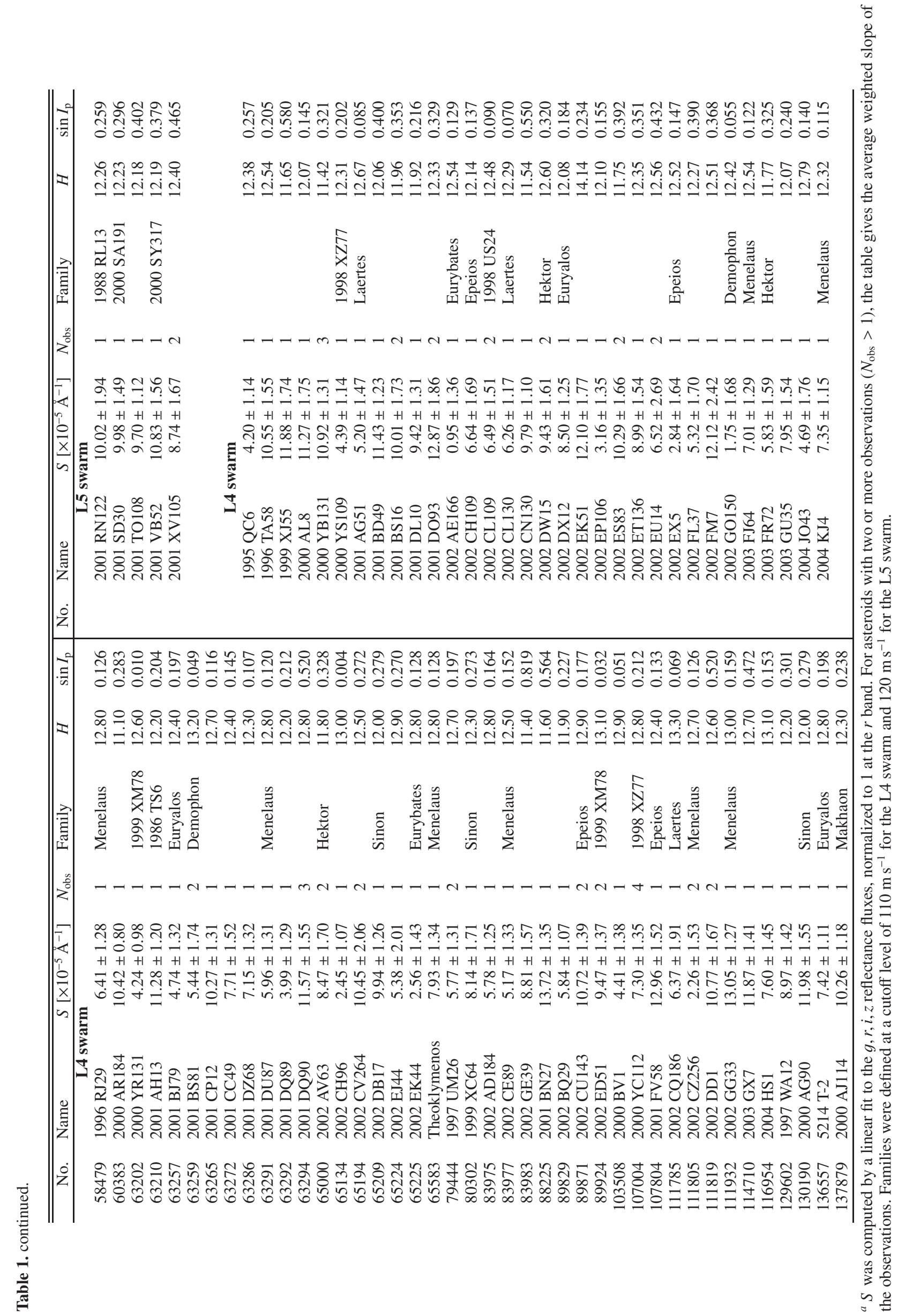




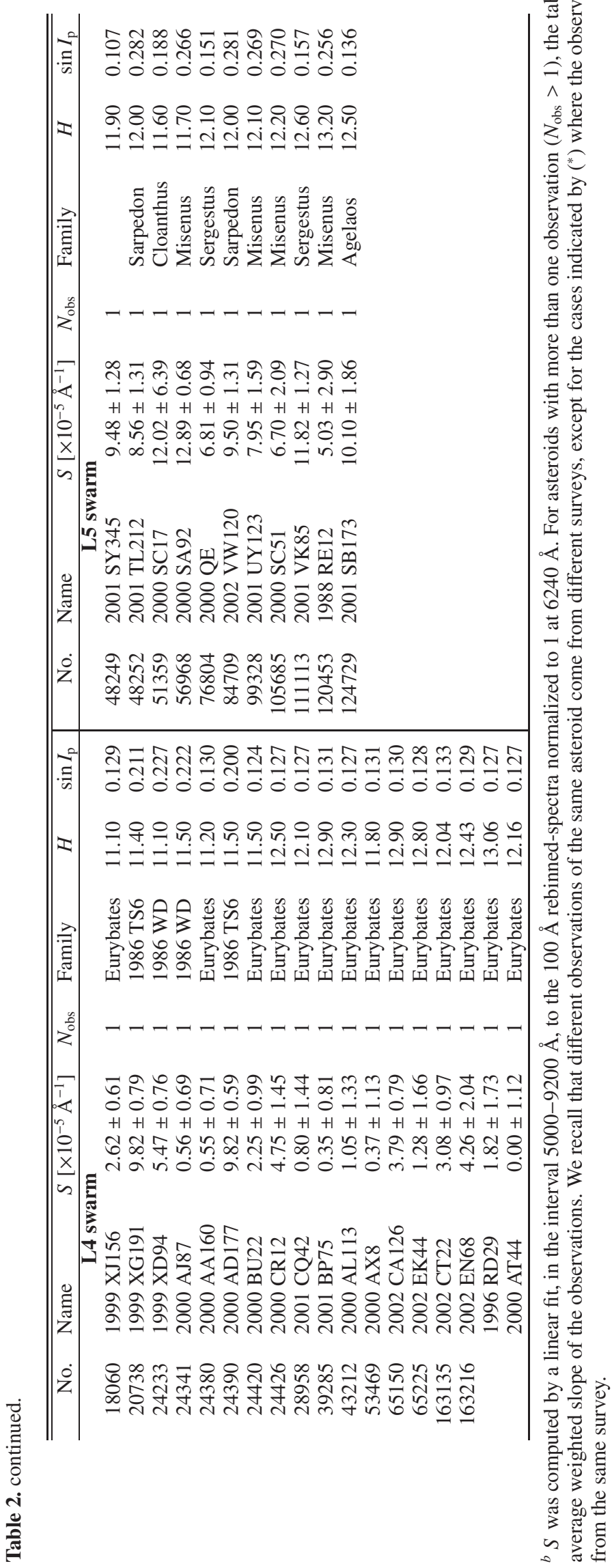




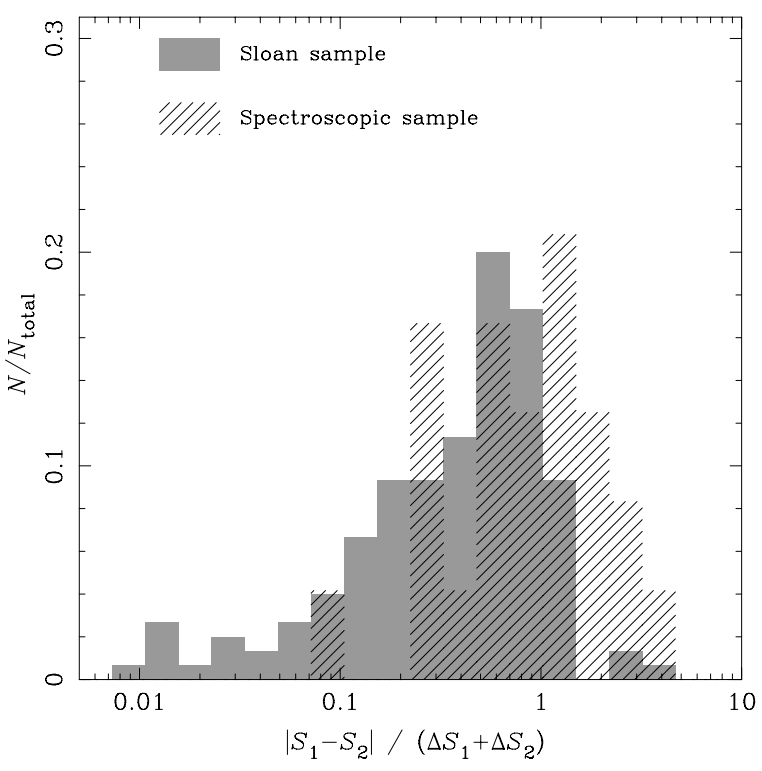

Fig. 2. Distribution of the parameter $\epsilon$ (see text) for the Sloan sample (gray histogram) and for the Spectroscopic sample (hatched histogram). Each histogram has been normalized such that its area is 1 .

where $S_{i}$ are the spectral slopes of two different observations of that asteroid and $\Delta S_{i}$ the corresponding errors. A value of $\epsilon<1$ indicates that these two observations are self-consistent, since their differences are within the individual errors. We can apply the same procedure to each asteroid with $N_{\text {obs }} \geq 2$ in Table 2, and compare the results. In Fig. 2, we show the distribution of $\epsilon$ values for the Sloan and Spectroscopic samples. The Sloan sample shows a good self-consistency among the observations of each asteroid with $N_{\mathrm{obs}} \geq 2$. Since, in most cases, the individual errors of the Sloan slopes are $\sim 10 \%$, this result supports the idea of a quite homogeneous sample.

On the other hand, a significant fraction of the Spectroscopic sample shows differences among the observations of each asteroid with $N_{\text {obs }} \geq 2$ that are larger than their errors. This may be explained by different observational conditions, different instrumental setup, and different reduction processes among the surveys. Another source for these differences could be related to surface composition heterogenity producing different spectra for different rotational phases of the body. This, for example, may be the case for asteroids (13463) Antiphos and (15535) 2000 AT177, observed by Dotto et al. (2006), and for (18493) 1996 HV9, observed by Fornasier et al. (2004) (see Table 2). The estimated errors of the Spectroscopic slopes are usually larger than $10 \%$, so the result shown in Fig. 2 supports the idea that the Spectroscopic sample is less homogeneous than the Sloan sample, as expected.

\section{Global distribution of spectral slopes}

In this section we analyze the distribution of spectral slopes of the whole population of known Trojan asteroids included in our data samples, with particular attention to the asteroid families. First, we compare the Sloan and the Spectroscopic samples, and then we discuss each sample separately.

\subsection{Comparisons between the samples}

In Fig. 3a, we show the distribution of Sloan slopes (349 observations) compared to the distribution of Spectroscopic slopes
(138 observations). The distribution of Sloan slopes shows a clear bimodality that is related to the presence of two different taxonomic types among the Jupiter Trojans: (i) the D-type, with spectral slopes $S \gtrsim 7.5 \times 10^{-5} \AA^{-1}$, corresponding to redder surfaces, and (ii) the P-type, with slopes $1.5 \lesssim S \lesssim 7.5 \times 10^{-5} \AA^{-1}$, corresponding to less reddish colors. There are also a small amount of observations compatible with the C-type taxonomy, with slopes $S \lesssim 1.5 \times 10^{-5} \AA^{-1}$, which correspond to more neutral colors.

The limiting slopes between the three taxonomic classes mentioned above are estimated within a $\pm 0.8 \times 10^{-5} \AA^{-1}$ interval of tolerance, which is the approximate bin size in Fig. 3a. It is worth stressing that these limiting slopes are totally arbitrary, and are not compatible with the convention adopted in the usual taxonomies, where the division between the P- and D-types should happen at $S \sim 5.5 \times 10^{-5} \AA^{-1}$ (e.g., Gil-Hutton \& Brunini 2008). Nevertheless, our choice is based on the natural separation of the slopes induced by the bimodality in their distribution, and it is valid as far as no mineralogical constraint is known to define the $\mathrm{P}$ and $\mathrm{D}$ taxonomic classes. We must recall that the bimodality of the Sloan sample has also been reported by Szabó et al. (2007) from the analysis of the SDSS-MOC3 colors. In fact, the principal color $t_{\mathrm{c}}^{*}$ introduced by these authors is strongly correlated to the spectral slope $S$.

On the other hand, the bimodality in the distribution of Spectroscopic slopes is less evident, although the three taxonomic classes still appear searated by the same limiting slopes. Quite notorious is the peak of C-type asteroids with $S \lesssim 2.0 \times$ $10^{-5} \AA^{-1}$, which is related to the observations of several members of a single asteroid family in L4. The presence of two peaks among the P-type asteroids, one around $S \sim 4.0 \times 10^{-5} \AA^{-1}$ and the other around $S \sim 6.5 \times 10^{-5} \AA^{-1}$, is also interesting. These two peaks might also be related to observations of specific families.

From Fig. 3a we may conclude that the D-type observations dominate the P-type in the approximate proportion 7:3. However, the Spectroscopic sample shows a larger abundance of P-type observations, relative to D-type, than the Sloan sample shows. This overabundance is explained below in terms of the observations of asteroid families. The Sloan slopes also appear more tightly clustered than the Spectroscopic slopes, which may be due to the smaller degree of homogeneity of the Spectroscopic sample. In spite of this, the Sloan slopes appear well correlated to the Spectroscopic slopes, as shown in Fig. 3b, for the few observations corresponding to asteroids included in both samples.

Since the aim of this work is to analyze the distribution of spectral slopes of the asteroid families, we proceeded to identify the different families in each Trojan swarm. We used the catalog of 1702 Trojan asteroids with known resonant proper elements maintained by the PETrA Project (Beaugé \& Roig 2001), and applied to this catalog the hierarchical clustering method (HCM, Zappalà et al. 1995). The mutual distance between any pair of asteroids in the proper elements space was computed according to the metric,

$d=\left[\frac{1}{4}\left(\frac{\delta a}{a_{0}}\right)^{2}+2(\delta e)^{2}+2(\delta \sin I)^{2}\right]^{1 / 2}$

(Milani 1993), where $\delta a, \delta e$, and $\delta \sin I$ are the differences in proper semi-major axis, proper eccentricity, and proper sinus of inclination, respectively, between the given pair of asteroids, and $a_{0}=5.2026 \mathrm{AU}$ is the average proper semi-major axis of the 

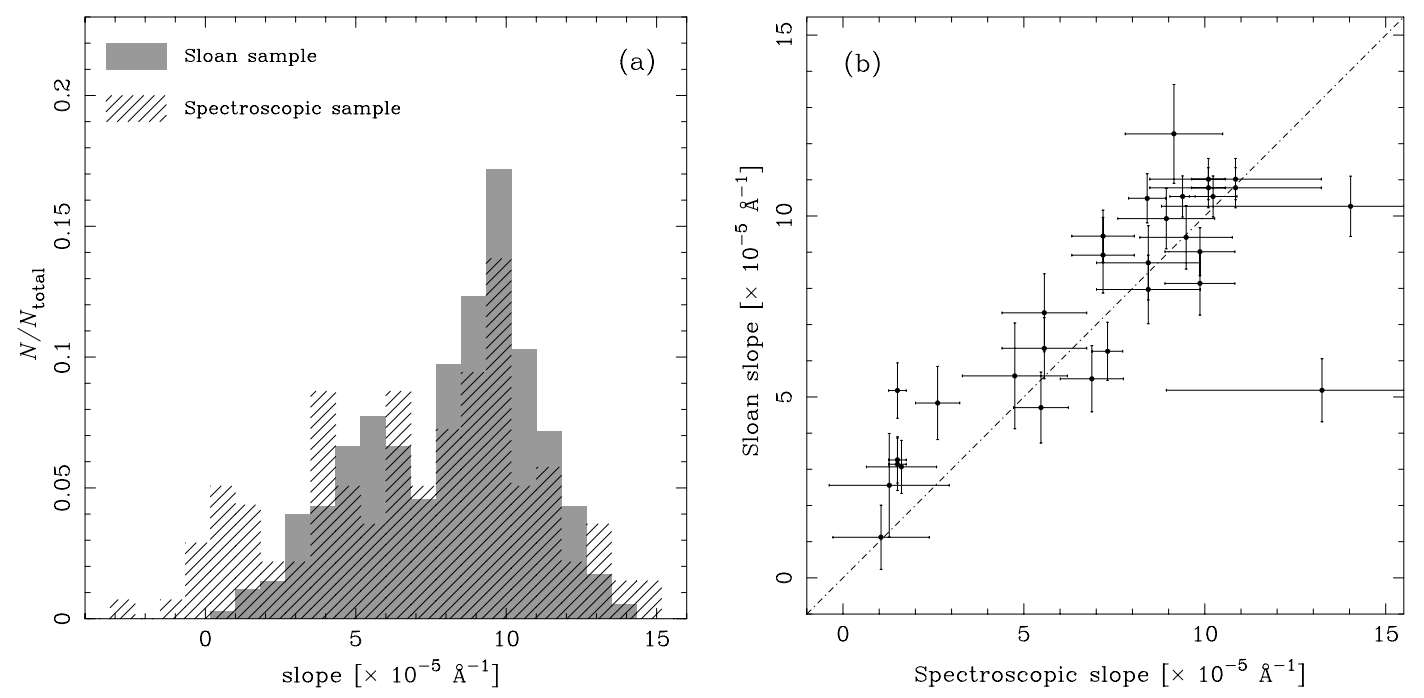

Fig. 3. a) Distribution of spectral slopes from the Sloan sample ( 349 observations, gray histogram) and from the Spectroscopic sample (138 observations, hatched histogram). Each histogram has been normalized such that its area is 1 . The distribution of Sloan slopes shows a clear bimodality related to the presence of two taxonomic types: P-type (smaller slopes) and D-type (larger slopes). This bimodality is less evident, but still appreciable, in the distribution of Spectroscopic slopes. The peak of Spectroscopic slopes around 0 is mostly caused by the observations of the Eurybates family by Fornasier et al. (2007). b) Comparison between the Spectroscopic slopes and the Sloan slopes from observations of asteroids included in both samples.
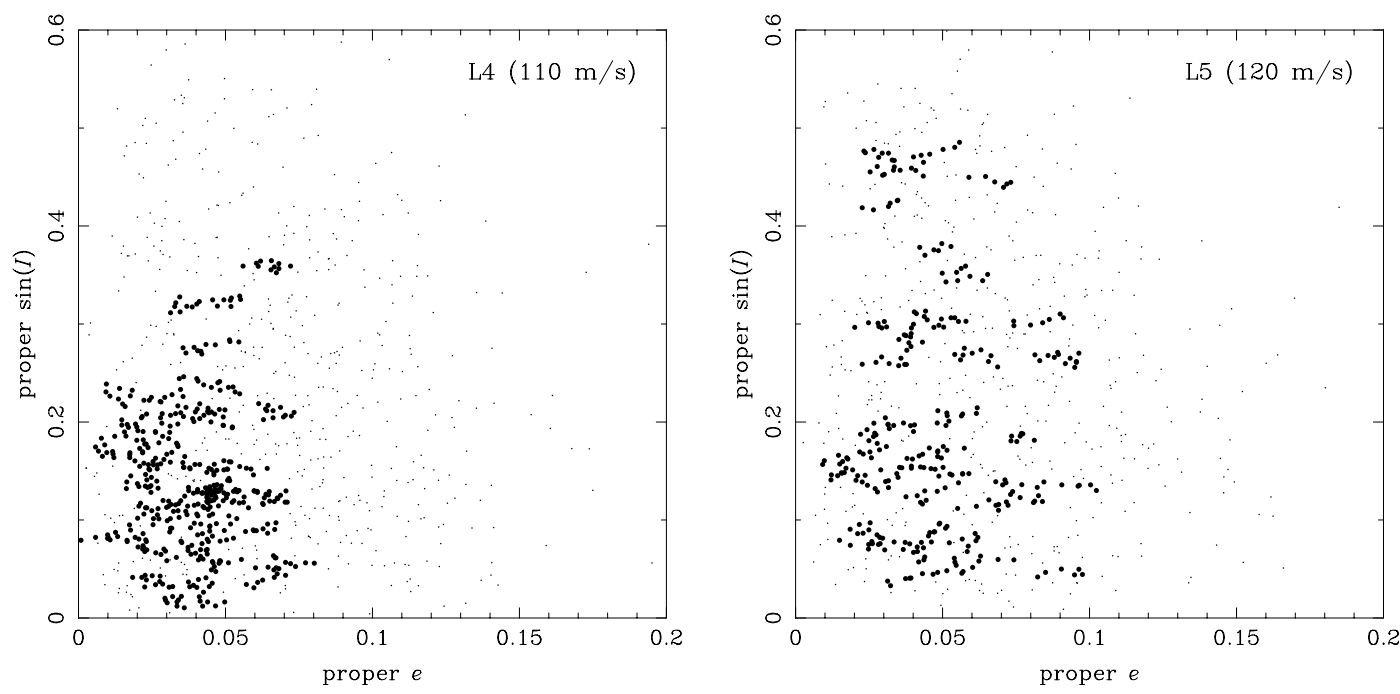

Fig. 4. Left panel: asteroid families (big dots) identified in the L4 swarm, projected in the space of proper eccentricity and inclination. Background asteroids are represented by small dots. The cutoff level is $110 \mathrm{~m} \mathrm{~s}^{-1}$. Right panel: the same, but for the L5 swarm. The cutoff level is $120 \mathrm{~m} \mathrm{~s}{ }^{-1}$.

Trojan population. Those bodies for which $d \leq d_{\text {cut }}$ were clustered together to form the families. The cutoff value $d_{\text {cut }}$ was chosen to be $110 \mathrm{~m} \mathrm{~s}^{-1}$ for the L4 swarm and $120 \mathrm{~m} \mathrm{~s}^{-1}$ for the L5 swarm, which are comparable to the corresponding quasirandom level of each swarm ${ }^{1}$. We have verified that values of $d_{\text {cut }}$ within $\pm 5 \mathrm{~m} \mathrm{~s}^{-1}$ around the above values produce practically the same results. Clusters with less than 8 members in the L4 swarm and with less than 6 members in the L5 swarm were considered statistical fluctuations and were disregarded. For a detailed explanation on the definition of $d_{\text {cut }}$ and the application of the HCM to the Trojan case refer to Beaugé \& Roig (2001). The distribution of the detected families in the space of proper eccentricity and inclination is shown in Fig. 4.

In Fig. 5, we show the slope distribution of family members (panel a) compared to the background asteroids (panel b).

\footnotetext{
1 The quasi-random level is the maximum level of statistical significance of the HCM.
}

The gray histograms correspond to the Sloan sample, while the hatched histograms correspond to the Spectroscopic sample.

The distribution of Sloan slopes of family members is comparable to the distribution of Spectroscopic slopes, except for the peak of Spectroscopic slopes around 0 in Fig. 5a, which correspond to the members of a very peculiar family in L4 (the Eurybates family; Fornasier et al. 2007). Both distributions show a clear bimodality, indicating the presence of the $\mathrm{D}$ and $\mathrm{P}$ taxonomic classes in the approximate proportion 6:4, respectively. It is worth recalling that the Sloan sample contains only $\sim 1.5$ times more observations of family members than the Spectroscopic sample, therefore, the agreement between the two samples is quite significant.

A bimodality is also observed among the background (nonfamily) asteroids, although in this case the D-type observations dominate over the P-type ones. This is clearly seen in the distribution of Sloan slopes, where the D and P classes appear in the 

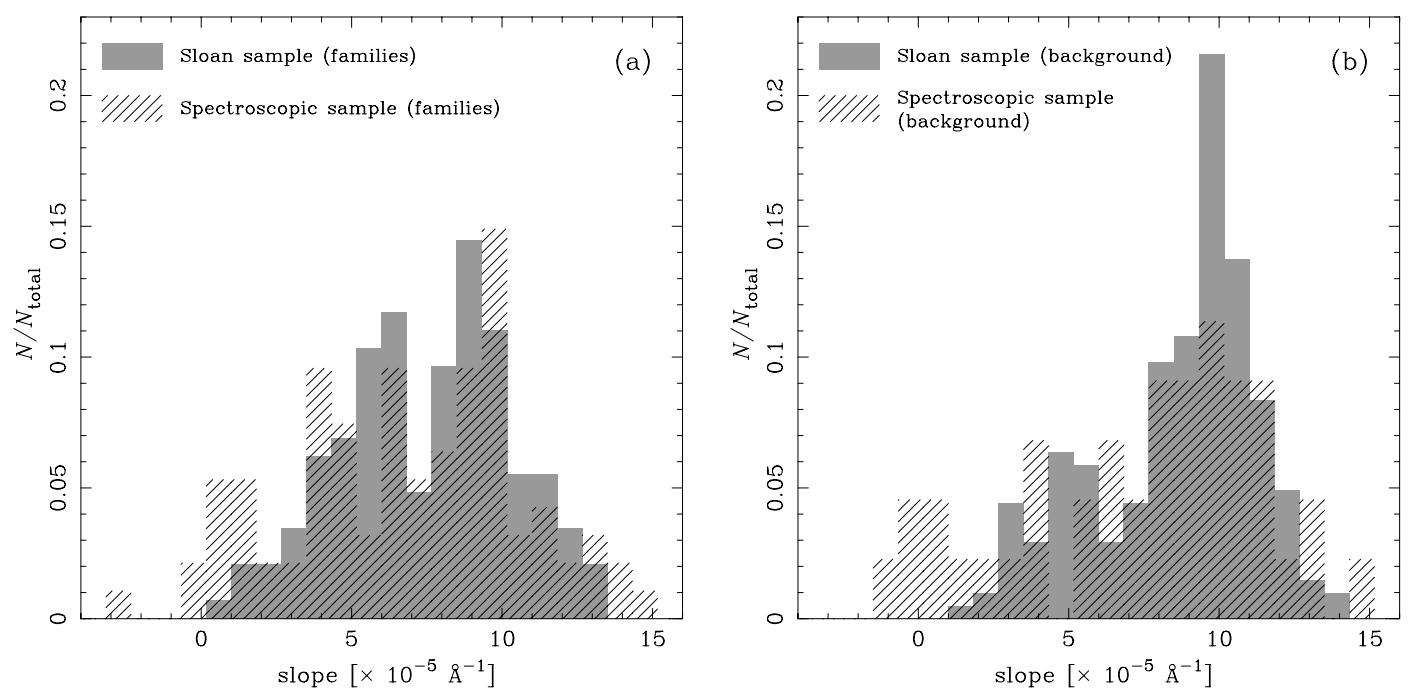

Fig. 5. a) Distribution of spectral slopes of observations corresponding to family members. The gray histogram correspond to the Sloan slopes and the hatched histogram to the Spectroscopic slopes. b) Same as a), but for the observations corresponding to background asteroids. Each histogram has been normalized such that its area is 1 .
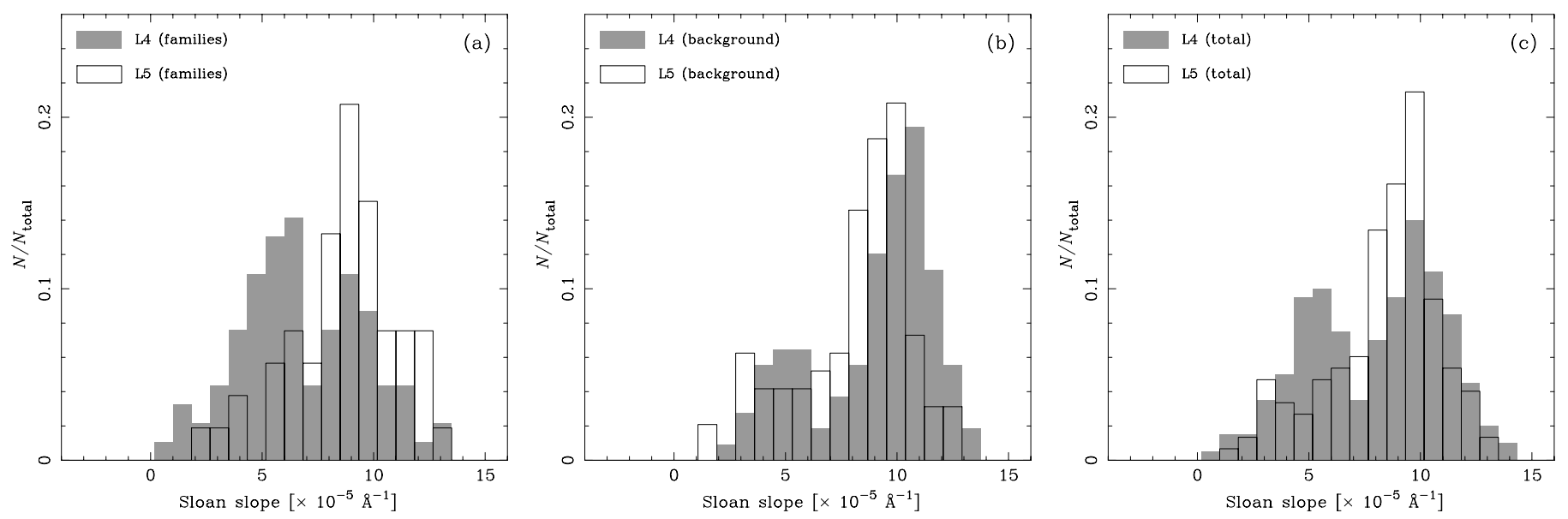

Fig. 6. a) Distribution of the Sloan slopes of family members only. The gray histogram corresponds to the L4 swarm and the outlined histogram to the L5 swarm. b) Same as a), but for the background asteroids only. c) Same as a), but for both family members and background asteroids together. Each histogram has been normalized such that its area is 1.

approximate proportion 8:2. A similar predominance, in the approximate proportion 7:3, is observed among the Spectroscopic slopes. However, the Sloan sample contains $\sim 4.5$ times more observations of background asteroids than the Spectroscopic sample, and it also goes much deeper in absolute magnitude. Therefore, the distribution of Sloan slopes seems to be more statistically significant than the distribution of Spectroscopic slopes. The lack of observations with Spectroscopic slopes around $S \sim 5.5 \times 10^{-5} \AA^{-1}$ may be the reason that led other authors to adopt this limit to separate the $\mathrm{P}$ and $\mathrm{D}$ taxonomic classes instead of the value $S \sim 7.5 \times 10^{-5} \AA^{-1}$ adopted here.

The above results indicate that the background is clearly dominated by reddish objects, while the families show a significant fraction of less reddish asteroids. This means that family members contribute a significant amount of the P-type asteroids found among the Trojan swarms. It also means that the families appear to be bluer, on average, than the background. Within this scenario, the different proportions of P-type relative to D-type observations between the two samples, shown in Fig. 3a, is simply due to the fact that families represent $70 \%$ of the observations in the Spectroscopic sample, but only $40 \%$ of the observations in the Sloan sample.

\subsection{Global analysis of the Sloan sample}

It is well known that asteroid families do not appear equally distributed among the L4 and L5 swarms. While the families in L4 are more conspicuous and tend to form large clusters, the families in L5 are smaller and tighter. The total number of families is also larger in L4 than in L5. Thus, it is interesting to analyze the distribution of Sloan slopes separately in each swarm. Figure 6a shows the distribution of Sloan slopes of family members in the L4 swarm (gray histogram) and in the L5 swarm (outlined histogram). The difference between the swarms is notorious. While in L4 the Sloan slopes show a predominance of P-type asteroids among the families, the L5 families appear dominated by D-type asteroids. The families in L5 are significantly redder than those in L4. On the other hand, the slope distribution of background asteroids, shown in Fig. 6b, is almost the same in the two swarms, with a significant peak of D-type asteroids. 

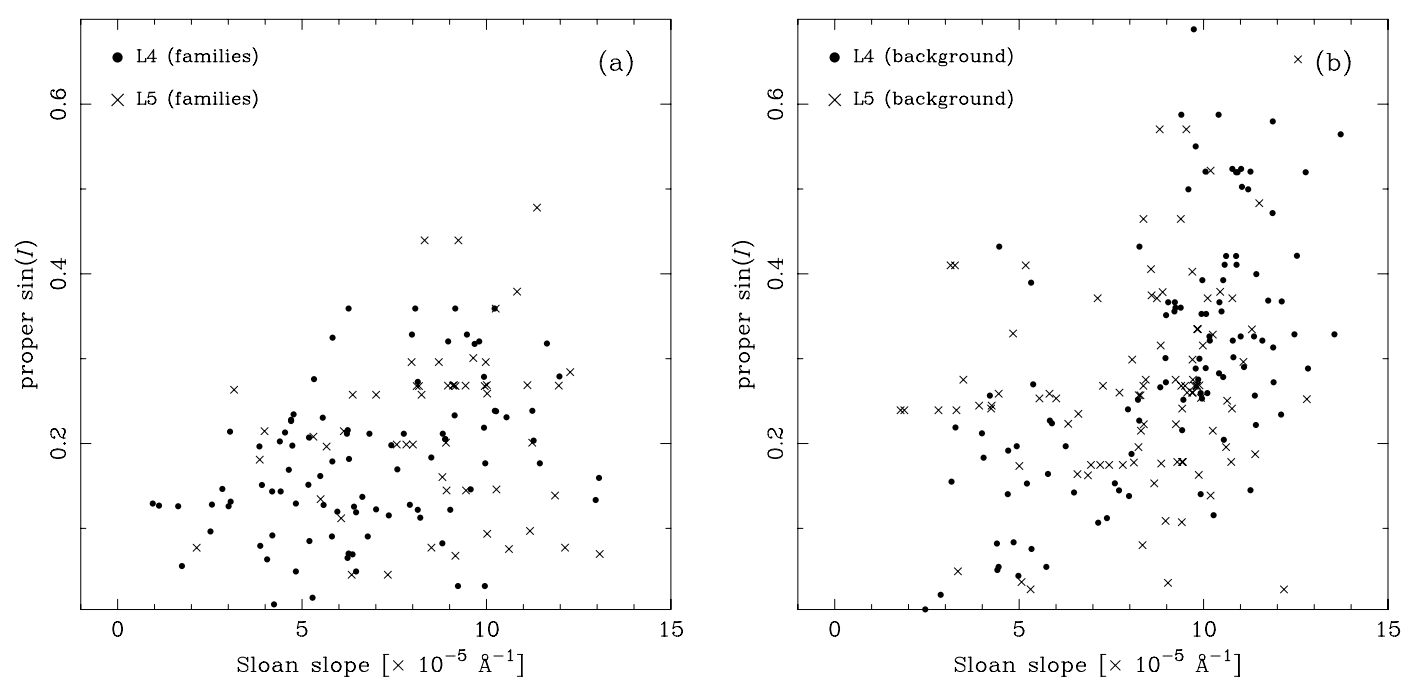

Fig. 7. a) Distribution of the Sloan slopes of family members as a function of proper inclination. Dots correspond to the L4 swarm and crosses to the L5 swarm. b) Same as a), but for the background asteroids. Note the significant lack of high-inclination background asteroids with small slopes (P-types).

The behavior observed in Fig. 6a, b may explain the different color distributions between the L4 and L5 swarms reported by Szabó et al. (2007) from the analysis of SDSS-MOC3 colors. These authors pointed out that the amount of redder asteroids (higher slopes), relative to the bluer ones (smaller slopes), is much larger in the L5 swarm than in the L4 swarm. The situation is clearly illustrated in Fig. 6c. They explained this difference on the basis of an observational selection effect that causes the detection of more asteroids with high orbital inclination, relative to those with low orbital inclination, in the L5 swarm compared to the L4 swarm. Since there is a clear correlation between color and orbital inclination, such that the bluer bodies have low inclinations while the redder ones are predominantly found at high inclinations, and since this correlation appears to be the same in both swarms, Szabó et al. (2007) conclude that it is natural to find a large fraction of redder bodies in the L5 swarm. The authors tried to overcome the observational selection effect by separating their observations into those corresponding to high-inclination asteroids $\left(>10^{\circ}\right)$ and those corresponding to low-inclination bodies $\left(<10^{\circ}\right)$, and showing that, with this separation, the differences between L4 and L5 almost disappear.

We believe, however, that a separation in terms of asteroid families and background asteroids, instead of orbital inclinations, provides a much better explanation, since it is clear from Fig. 6 that the swarms differ in their color distributions due to the presence of the asteroid families. The advantage of this scenario is that it has a physical basis and does not require the invocation of any strange observational bias. It is also interesting to analyze the color-inclination correlation in terms of asteroid families. In Fig. 7 we show the distribution of asteroid family members (panel a) and background asteroids (panel b) in the plane of spectral slope vs. orbital inclination. Dots and crosses represent the L4 and L5 observations, respectively. The family members do not show any apparent correlation between color and inclination in contrast with the background which is strongly correlated. This correlation appears to be the same in both swarms, as Szabó et al. (2007) conjectured.

We must note that the separation in low- and high-inclination populations proposed by Szabó et al. (2007) partially works to explain the different color distributions between L4 and L5 because the family members are not uniformly distributed in terms of proper inclination. In fact, the families in the L4 swarm are mostly concentrated at low inclinations, while the families in L5 spread over a wider range of proper inclinations, as we can see in Fig. 4. If we consider only the high inclination asteroids ( $\sin I \gtrsim 0.2$ ), then the L4 swarm is dominated by background asteroids (Fig. 4), which are predominantly red (Fig. 7b). The L5 swarm has a larger proportion of asteroid families at high inclinations (Fig. 4), but these are also predominantly red (Fig. 6a) like the background. Thus, both swarms show the same color distribution at large inclinations. On the other hand, if we consider the low-inclination asteroids ( $\sin I \lesssim 0.2)$, the asteroid families significantly contribute to the slope distribution. While the background tends to be bluer (Fig. 7b), the families cover a wider range of colors (Fig. 6a), and this tends to disguise the differences in slope distribution between the swarms. This is precisely the result found by Szabó et al. (2007).

Another interesting result concerns the correlation between spectral slope and absolute magnitude (or size). Figure 8 is analogous to Fig. 7, but in terms of absolute magnitude instead of orbital inclination. If we eliminate the few large bodies $(H \lesssim 9)$ from the sample, then the families (Fig. 8a) do not show any apparent correlation, but the background asteroids (Fig. 8b) shows a weak correlation since bodies in the range $9 \lessgtr H \lesssim 11$ are predominantly red. Note that if we consider the families and the background together, the slope-size correlation is disguised and this is probably the reason why Szabó et al. (2007) did not detected this correlation in their analysis.

The results of Figs. $7 \mathrm{~b}$ and $8 \mathrm{~b}$ led us to conclude that large background asteroids in both Trojan swarms tend to be redder and tend to be located at large orbital inclinations.

\subsection{Global analysis of the Spectroscopic sample}

The behavior observed in Figs. 6-8 is not clearly reproduced by the Spectroscopic sample. There are two main reasons for this: (i) about $70 \%$ of the Spectroscopic sample is constituted of observations of asteroids that are members of specific preselected asteroid families, and (ii) the observations of background asteroids are limited to the largest Trojans only. These selection effects are expected to introduce an important bias with respect to the Sloan sample, where the observed family members were 

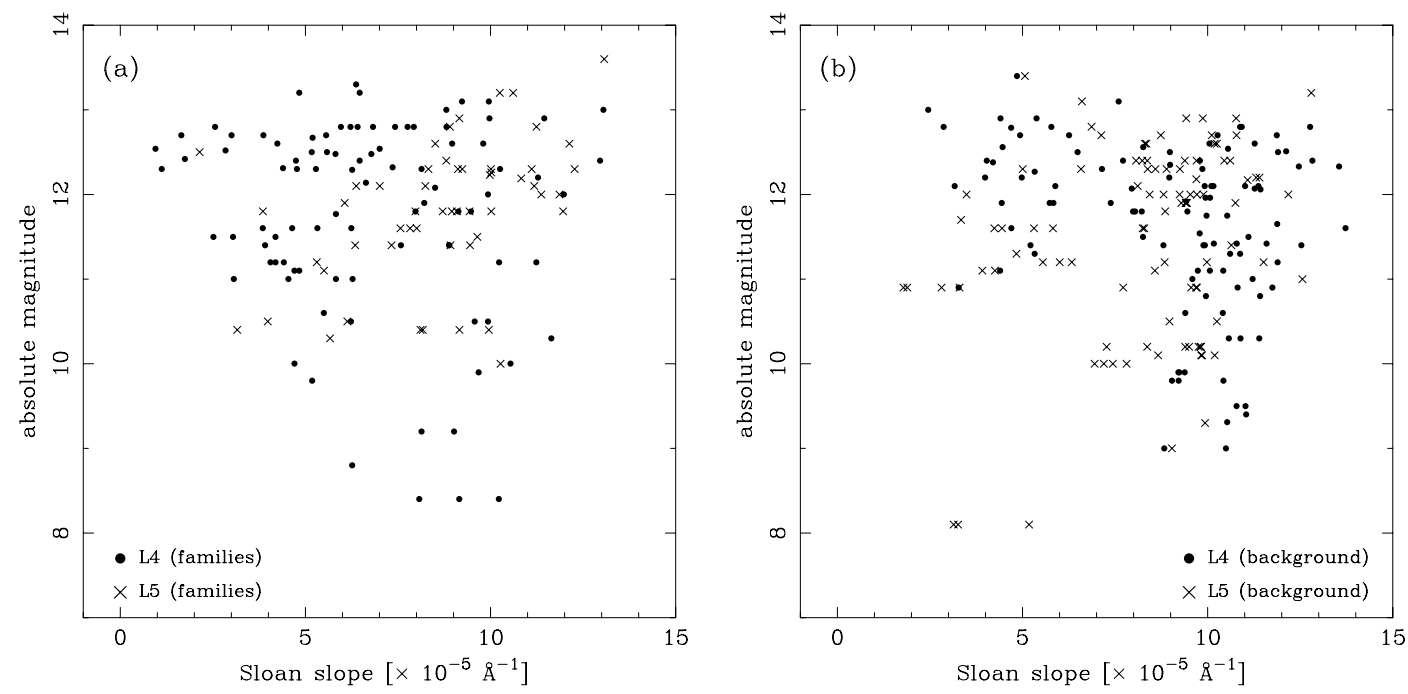

Fig. 8. a) Distribution of Sloan slopes of family members as a function of absolute magnitude. Dots correspond to the L4 swarm and cross to the L5 swarm. b) Same as a), but for the background asteroids. Note the significant lack of large background asteroids with small slopes (P-types).
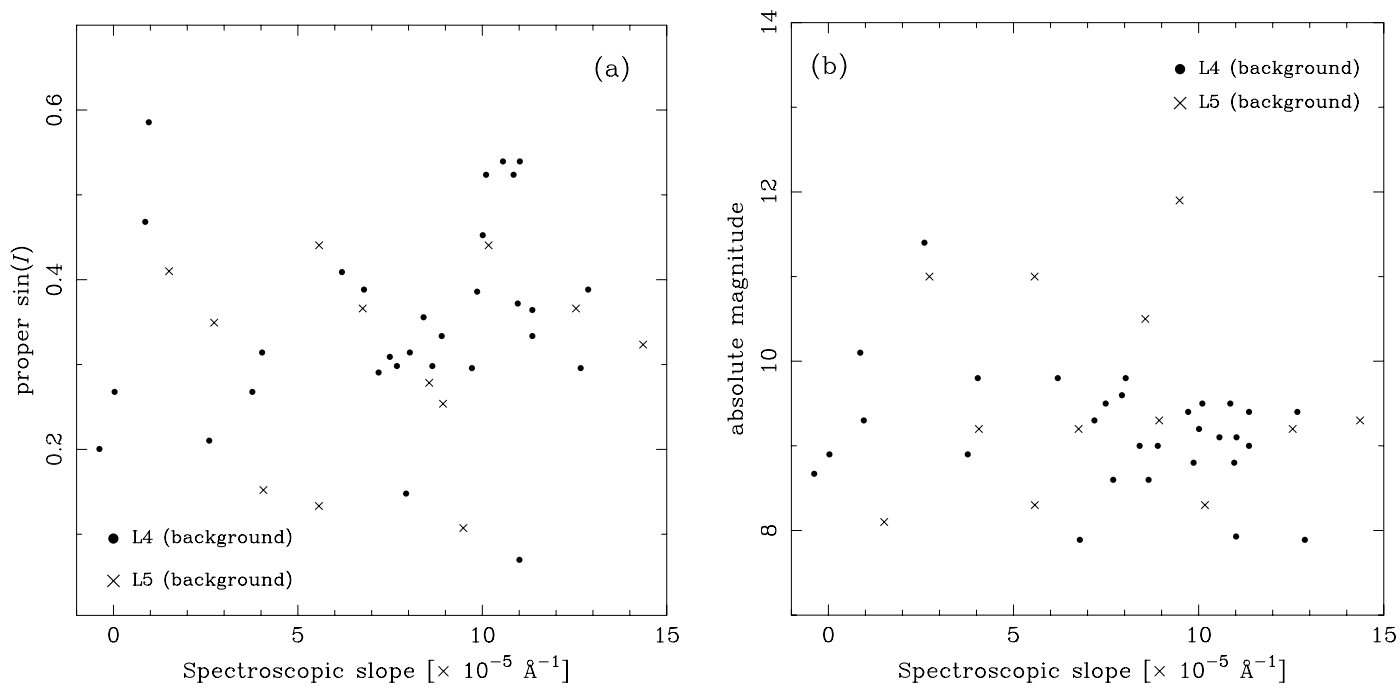

Fig. 9. Distribution of the Spectroscopic slopes of background asteroids as a function of proper inclination a) and absolute magnitude b). Dots correspond to the L4 swarm and cross to the L5 swarm.

"chosen at random", and the amount of small background asteroids observed is much larger.

Nevertheless, the relations between slope, inclination, and size among the Spectroscopic sample reveal certain features that are compatible with the correlations observed in the Sloan sample. Of particular interest are the distributions of spectral slope, proper inclination, and absolute magnitude of the background asteroids in the Spectroscopic sample. These distributions are shown in Fig. 9. The concentration of reddish objects (D-type) at high inclinations and low absolute magnitude is particularly notorious and confirms our finding that large background Trojans in both swarms tend to be redder and tend to have large inclinations.

An analysis of a larger data set of spectroscopic data has been performed by Fornasier et al. (2007). Their sample includes the same observations that we include in our Spectroscopic sample, plus other spectroscopic observations from Jewitt \& Luu (1990) and Fitzsimmons et al. (1994), totaling 142 different Trojan asteroids. Fornasier et al. (2007) found that the L4 swarm appears to have a larger fraction of P-type asteroids, relative to D-type, compared to the L5 swarm. They did not find any slope-size correlation, although they detected that the distribution of spectral slopes is narrower at large sizes. This implies a slight predominance of D-type asteroids among the large Trojans $(50 \lesssim D \lesssim 120 \mathrm{~km}$ ), which is in line with the situation seen in Figs. $8 \mathrm{a}, \mathrm{b}$ and in Fig. $9 \mathrm{~b}$ for $9 \lessgtr H \lesssim 11$.

\subsection{Discussion}

The existence of the correlations shown in Figs. $7 \mathrm{~b}$ and $8 \mathrm{~b}$ points to another correlation between inclination and size among the background asteroids. The relation between these two quantities is shown in Fig. 10 for all the known background Trojans. Although a correlation appears to be evident, this plot must be analyzed carefully. First, the population of known Trojans is complete only up to $H \sim 12$ (e.g. Szabó et al. 2007), and the lack of high inclination background asteroids with $H \gtrsim 12$ is most probably an artifact of the incompleteness of the sample. On the other hand, the relative lack of large background asteroids $(H \lesssim 10)$ with small inclinations is a real effect, and reinforces the results presented in Figs. 7b, 8b, and 9. It is worth 
recalling that Szabó et al. (2007), analyzing a large sample of candidate Trojans, did not find any correlation between size and inclination. However, these authors were not able to analyze the background separately from the families, and moreover they had to use the latitude with respect to Jupiter's orbit as a proxy for the orbital inclination. Since the families contribute significantly to the low-inclination Trojan population and the latitude represents a lower limit of the orbital inclination, the behavior shown in Fig. 10 could have never been reproduced by Szabó et al.

The fact that only the background asteroid show correlations between spectral slope, absolute magnitude, and orbital inclination, and that the correlations are similar in both the L4 and L5 swarms, may put important constraints to the origin and evolution of Jupiter Trojans. No dynamical mechanism among the Trojans is known to favor the evolution of asteroids according to their size or to their surface physical properties ${ }^{2}$. Therefore, these correlations may have a primordial origin. Alternatively, the correlations may be the by-product of collisional evolution. We speculate here about two possible scenarios.

One scenario involves the idea that the $\mathrm{P}$ and $\mathrm{D}$ classes are related to different mineralogies and, consequently, to different material strengths. Let us assume that P-type asteroids are easier to break up than D-type asteroids. Recall that this is just an assumption and there is no evidence, neither observational nor theoretical, to support it. Therefore, large P-type asteroids will tend to fragment in smaller bodies, while large D-type asteroids will tend to remain intact, causing a loss of large P-type asteroids as suggested in Fig. 8b. In addition, fragments from P-type asteroids may acquire larger ejection velocities after a break up than fragments from D-type asteroids. Since the islands of stability around L4 and L5 shrink at large inclinations (e.g., Marzari et al. 2003; Schwarz et al. 2004), many of these P-type fragments might be ejected beyond the stability limits of the swarms causing the lack of high-inclination P-type asteroids observed in Fig. 7b. The predominance of P-type asteroids among the L4 families is in line with this scenario but, on the other hand, the predominance of D-type asteroids among the L5 families is against it.

Another scenario involves the idea that the P and D classes represent the same mineralogy but modified by some aging process like space weathering. Let us assume that space weathering produces a reddening of the surfaces, so D-type asteroids have older surfaces than P-type asteroids. The surfaces may be renewed either by disruptive collisions that expose the "fresh" interior of the parent body, or by resurfacing collisions. We could expect that both collisional phenomena are more frequent at low inclinations than at high inclinations, and more frequent among the small bodies than among the large ones. Thus, high inclination and large asteroids would be, on average, older (i.e. redder) than low inclination and small ones, in agreement with Figs. 7b and $8 \mathrm{~b}$. This scenario would also imply that families in L5 are, on average, older than those in L4.

The above scenarios have several limitations because none of them are well constrained. The main limitations are:

- The mineralogy associated with the P- and D-types is totally unknown, and so is the corresponding material strengh. In fact, some authors claim that D-type asteroids would be more fragile than P-types (Dahlgren et al. 1997).

- The rate of collisional events that can produce disruption or resurfacing depending on diameter and orbital inclination is poorly constrained.

2 The Yarkovsky effect, which depends on size and surface properties, also depends on the Sun distance and it is negligible at $5 \mathrm{AU}$.

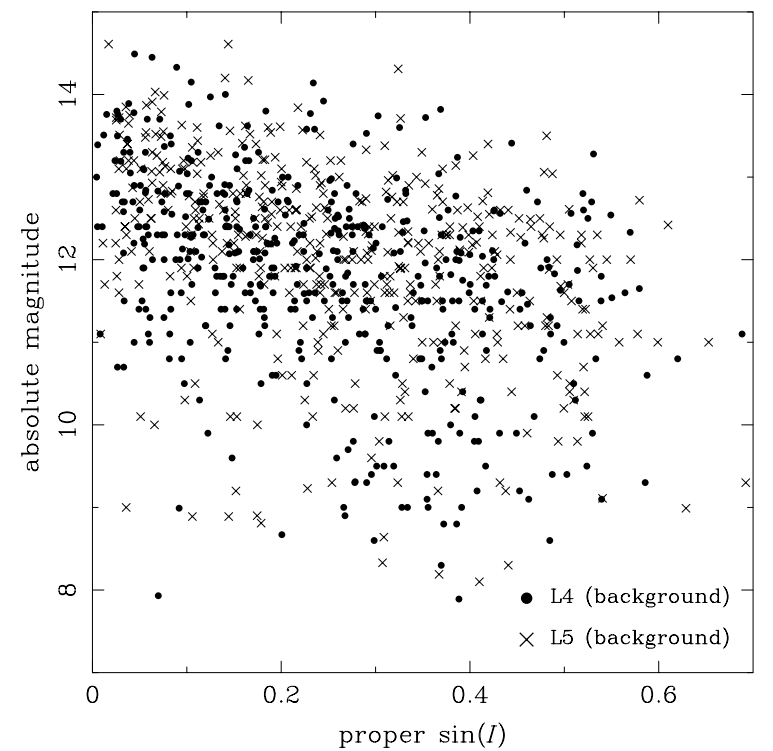

Fig. 10. Distribution of proper inclinations in terms of absolute magnitude for 913 known background Trojans. Dots correspond to the L4 swarm and cross to the L5 swarm. Note the relative lack of large asteroids with small inclinations.

- The actual effect of space weathering on spectrally featureless surfaces and the timescale to produce a significant change in the spectral slope are also unknown. Some authors propose that space weathering would tend to neutralize the colors of initially red surfaces (Moroz et al. 2004), so that P-type asteroids would have older surfaces than D-types. Another hypothesis proposes that space weathering may have two phases: an initial phase in which it produces a reddening of the surfaces up to a saturation level, and a second phase in which it produces the opposite effect, leading to more neutral color surfaces, together with a reduction of the overall albedo. Within this scenario, P-type asteroids could have surfaces that are either too young (high albedo) or too old (low albedo), while D-types would have mid-age surfaces. D-type asteroids would also be, on average, more numerous because an asteroid would spend "most of its life" showing a reddish surface, unless a collision modifies it. This might be causing the overall abundance of D-type asteroids among the Trojans. The knowledege of the albedo values for a large amount of Jupiter Trojans might help to better contrain this scenario. Unfortunately, up to now, very few Trojans have known albedos.

- Finally, what we observe might be the product of a complex combination of all these effects.

\section{Distribution of spectral slopes for selected asteroid families}

In the previous section, we discussed the global distribution of spectral slopes among Trojan asteroid families and background asteroids. In this section, we analyze some particular families, selected in view of their interest and the number of its members contained in both the Sloan and the Spectroscopic samples. For this analysis, we did not consider all the observations available in the samples. Instead, we used the slopes listed in Tables 1 and 2 (i.e., for asteroids with more than one observation we consider the average slope of the observations). 

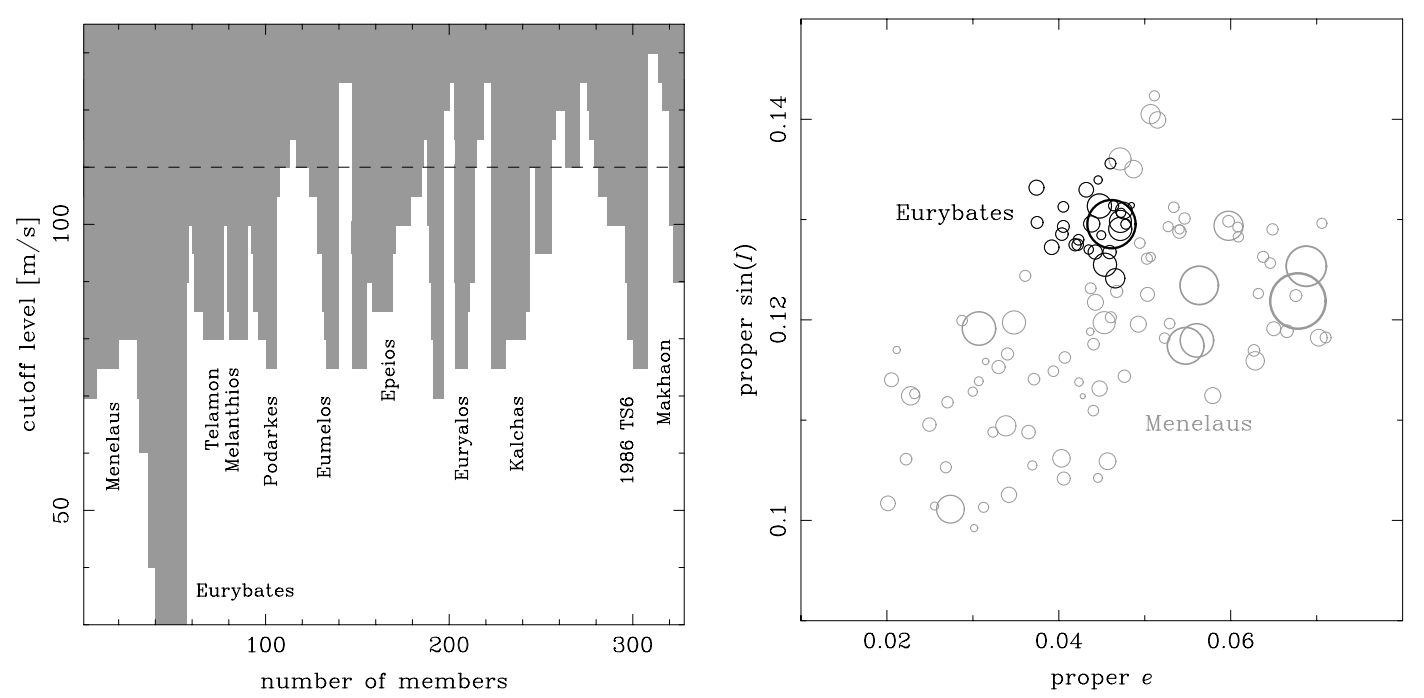

Fig. 11. Left panel: dendogram of the Menelaus clan, indicating the main families identified. The dashed horizontal line is the cutoff used in this study. Right panel: distribution in the space of proper elements of the Menelaus family (gray circles) as detected at $d_{\text {cut }}=110 \mathrm{~m} \mathrm{~s}^{-1}$, and of the Eurybates family (black circles) as detected at $d_{\text {cut }}=70 \mathrm{~m} \mathrm{~s}^{-1}$. The size of each circle is proportional to the asteroid size.

\subsection{Families in the L4 swarm}

\subsubsection{The Menelaus clan}

Several families in the L4 swarm merge together at high values of the cutoff $\left(d_{\text {cut }}>125 \mathrm{~m} \mathrm{~s}^{-1}\right)$ to form a big clan of families similar to the Flora clan in the inner asteroid Main Belt. This clan gets its name after the main member, asteroid (1647) Menelaus. The structure of the Menelaus clan is shown in Fig. 11 (left) in the form of a dendogram (Zappalà et al. 1995). Each stalactite in the dendogram represents a different family within the clan, and it is easy to see how the families are better resolved as we go to lower values of the cutoff. The word "clan" invokes some kind of common origin, but the fact that several families form a clan does not necessarily imply that they all come from the same ancestor. The taxonomic analysis of the clan members may help to better understand this problem.

As seen in Fig. 11, the more robust family of the clan is the Menelaus family itself, which counts more than 100 members at the quasi-random level $\left(\sim 104 \mathrm{~m} \mathrm{~s}^{-1}\right)$ and represents the largest family in the L4 swarm. The small families of Telamon, Melanthios and Podarkes separate from the Menelaus family at lower cutoffs, but they soon disappear. On the other hand, the Eurybates family appears as a robust cluster that survives down to small cutoffs. Indeed, the Eurybates family forms a tight cluster within the Menelaus family, as shown in Fig. 11 (right). From the sole analysis of Fig. 11, it is difficult to decide whether the Eurybates family is a subcluster of the Menelaus family - i.e., a family formed by the secondary breakup of a former Menelaus family member - or whether the Eurybates and Menelaus families are two different families that simply overlap in the space of proper elements.

The taxonomy of these two families has been analyzed by Dotto et al. (2006, hereafter D06) and by Fornasier et al. (2007, hereafter F07), who obtained spectra of 3 members of the Menelaus family and 17 members of the Eurybates family. These authors found that the Menelaus family is mostly a D-type family, but the Eurybates family is dominated by C-type asteroids. A slightly different result is obtained from the analysis of our data samples.

Figures $12 \mathrm{a}, \mathrm{b}$ show the spectral slopes of the Menelaus and Eurybates families as a function of the absolute magnitude.
At large sizes $(H<11$, which corresponds to $\sim 40 \mathrm{~km})$, the Menelaus family shows a slight predominance of D-type asteroids: (1749) Telamon; (5258) 1989 AU1; and (13362) 1998 UQ16. Asteroid (1647) Menelaus appears to be a P-type, but could be classified as D-type if we account for its error and recall that the limiting slope of $7.5 \times 10^{-5} \AA^{-1}$ between the P- and D-types has a $\pm 0.8 \times 10^{-5} \AA^{-1}$ uncertainty (actually, D06 classified this asteroid as D-type). Asteroid (5244) Amphilochos is the only one P-type asteroid with $H<11$. On the other hand, at the small sizes $(H>11)$ the family is clearly dominated by P-type asteroids.

The results for the Eurybates family (Fig. 12b) are in line with the findings of F07. Of the six asteroids contained in the Sloan sample, three asteroids - (9818) Eurymachos; (18060) 1999 XJ166; and (24426) 2000 CR12 - are P-type, and one asteroid - (43212) 2000 AL113- is C-type. These four asteroids were given the same taxonomic classification by F07. The fifth asteroid - (65225) 2002 EK44 - appears to be a P-type, but due to its error, can be classified as C-type in agreement with F07. The sixth body - 2002 AE166 - is a C-type asteroid and was not observed by F07.

Figures $12 \mathrm{c}-\mathrm{g}$ show the spectral slopes of other members of the Menelaus clan. The Epeios family (Fig. 12c) has not been previously observed by any spectroscopic survey, and so the Sloan slopes shown here provide the first taxonomic information about this family, which appears to be constituted mostly by P-type asteroids, especially at the large sizes.

The 1986 TS6 family (Fig. 12d) has been observed by D06 ${ }^{3}$ and F07. The available data, including the Sloan slopes, indicate that this family has two well-separated components, one P-type and one D-type, regardless of their body size. Note, however, that these two components cannot be resolved in terms of proper elements, i.e., the P- and D-type members are mixed in the same cluster even for the smallest possible cutoffs. The largest asteroid in the family, (5025) 1986 TS6, shows significantly different values of Sloan slope $\left(5 \times 10^{-5} \AA^{-1}\right)$ and Spectroscopic slope $\left(13 \times 10^{-5} \AA^{-1}\right)$, but since the latter has a large error arising from

\footnotetext{
3 These authors refer to it as the Makhaon family due to the large cutoff used, but it is clear from Fig. 11 that Makhaon is a different family. This has been correctly addressed by F07.
} 

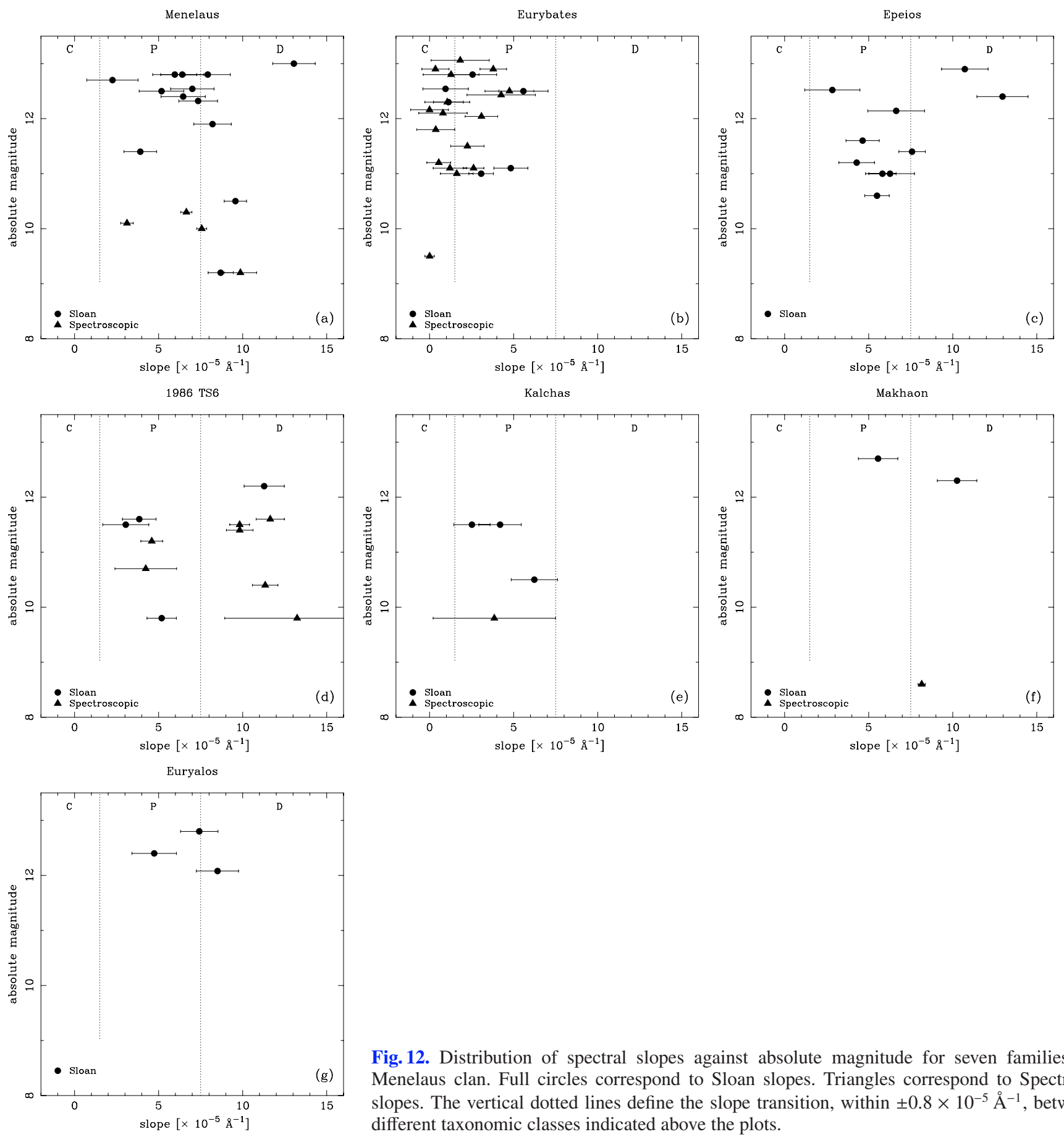

Fig. 12. Distribution of spectral slopes against absolute magnitude for seven families of the Menelaus clan. Full circles correspond to Sloan slopes. Triangles correspond to Spectroscopic slopes. The vertical dotted lines define the slope transition, within $\pm 0.8 \times 10^{-5} \AA^{-1}$, between the different taxonomic classes indicated above the plots.

a noisy spectrum of Bendjoya et al. (2004), its value should be considered with care.

Finally, the Kalchas family (Fig. 12e) appears to be a P-type family, while the Makhaon and Euryalos families (Figs. 12f, g) show a mixture of P- and D-type asteroids. However, the amount of spectral slopes for these three families is too small to draw definite conclusions about them.

The above results points to the idea that not only the Menelaus clan as a whole, but also the individual families are quite heterogeneous in terms of taxonomic classes, including from the reddest D-type asteroids to the neutral-color C-type ones. Note also that, among the families with the largest amount of observations, the spectral slopes do not show any systematic trend with size. In the case of the Menelaus and 1986 WD families, the larger the bodies the redder the slope. But the situation is inverted in the case of the Epeios family where the larger the bodies, the bluer the slope, and no trend at all is observed in the case of the 1986 TS6 family.

\subsubsection{Other families}

Figure 13 shows the distributions of spectral slopes in terms of absolute magnitude of four families that are not members of the Menelaus clan. The 1986 WD family (Fig. 13a) has been studied by D06 and F07, who found a wide range of slopes from the D- to the C-type. The Sloan slopes tend to confirm these findings. This family is small ( $\sim 15-20$ members $)$ and at cutoff values slightly smaller than the quasi-random level, it looses half of 

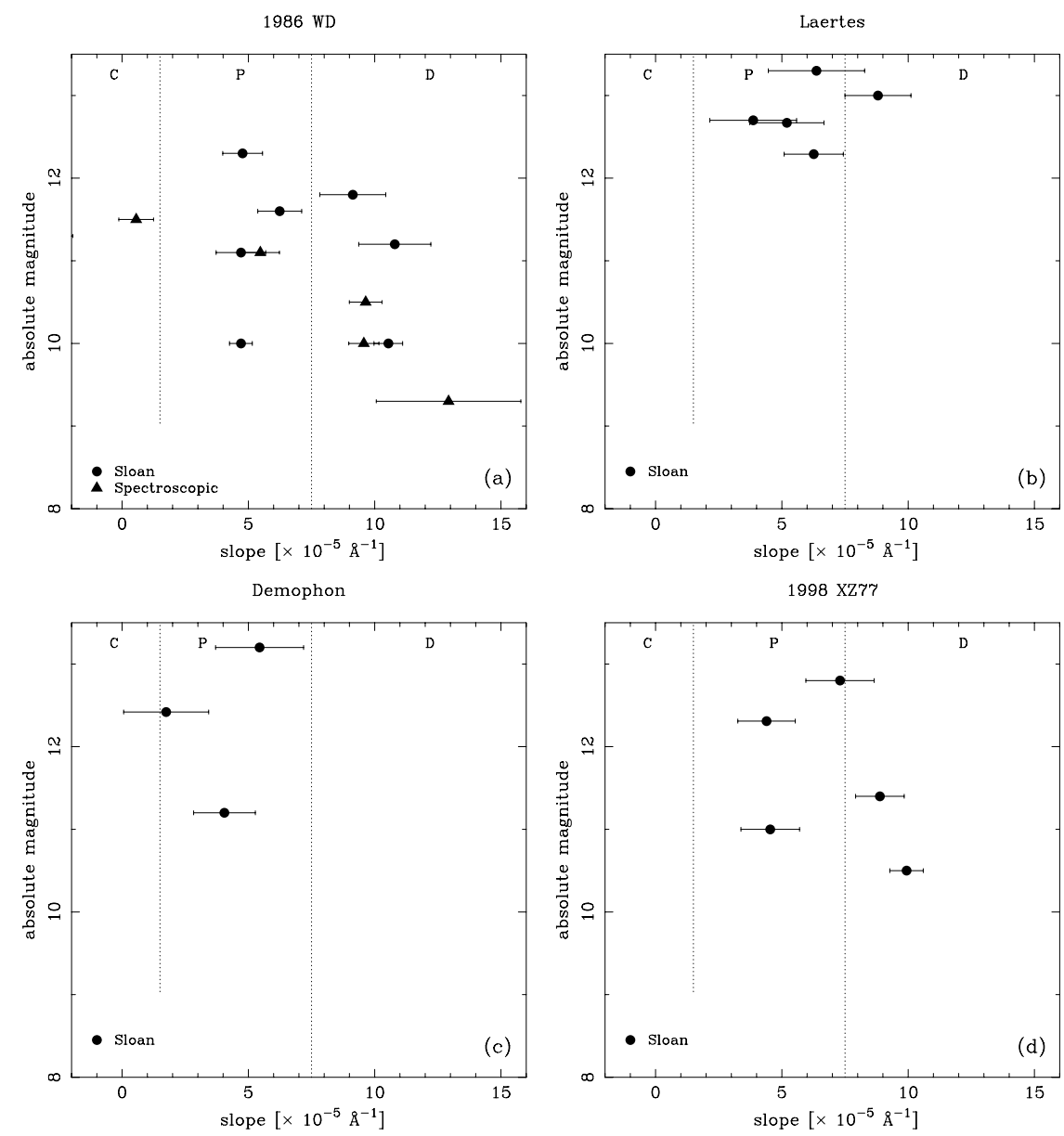

Fig. 13. Same as Fig. 12, but for four families in the L4 swarm.

its members. The family is no longer identified at cutoffs smaller than $95 \mathrm{~m} \mathrm{~s}^{-1}$. Therefore, the diversity of taxonomic classes may be related to a significant contamination of interlopers.

The case of the Laertes family (Fig. 13b) is somehow different. This family is also small ( $\sim 15-20$ members), but it survives down to cutoff $80 \mathrm{~m} \mathrm{~s}^{-1}$ losing only $40 \%$ of its members. All the members are small bodies $(H>11)$, including (11252) Laertes. The few members contained in the Sloan sample (the family has never been observed spectroscopically) show a quite homogeneous distribution of spectral slopes, all belonging to the P-type. Unfortunately, this family is located at a small inclination ( $\sin I \sim 0.08$ ), and it cannot be distinguished from the background also dominated by P-type asteroids. The identification of the Laertes family as a real P-type family relies more in the accuracy of the HCM than in the distribution of its spectral slopes.

The Demophon family (Fig. 13c) and the 1998 XZ77 family (Fig. 13d) are small clusters ( 7 and 12 members, respectively), which are not detected at cutoffs smaller than $110 \mathrm{~m} \mathrm{~s}^{-1}$. Both families have almost half of their members observed in the Sloan sample. The Demophon family shows a homogeneous P-type taxonomy, while the 1998 XZ77 family shows a mixture of Pand D-type asteroids. Since these two clusters do not survive at smaller cutoffs, it is difficult to determine whether they are authentic families or simple statistical fluctuations of the HCM.

Other interesting cases are the high-inclination families in the L4 swarm. There are only three of these families, with $\sin I>0.25$ : Hektor, Teucer, and Sinon. The distribution of the respective spectral slopes are shown in Fig. 14. Hektor and Sinon families (Figs. 14a, c) have not been observed by previous spectroscopic surveys, and the Sloan slopes provide the first clues about their taxonomic composition. The slopes in Fig. 14 point to a predominance of D-type asteroids, making these families indistinguishable from the background.

\subsection{Families in the L5 swarm}

\subsubsection{The Anchises clan}

The L5 swarm has its own clan of families, although it is somehow different from the Menelaus clan in L4. The Anchises clan, named after asteroid (1173) Anchises, is quite tight and constituted by only five families identified at $d_{\text {cut }}=120 \mathrm{~m} \mathrm{~s}^{-1}$ : Panthoos, Polydoros, Sergestus, Agelaos and 1999 RV165. All these families merge in the clan at $d_{\text {cut }}=150 \mathrm{~m} \mathrm{~s}^{-1}$. The taxonomic analysis of this clan indicates that it is populated by both $\mathrm{P}$ - and D-type asteroids, covering a wide range of spectral slopes. But at variance with the Menelaus clan, the individual families of the Anchises clan appear to be more homogeneous in terms of taxonomy.

The Panthoos family appear to be a P-type family (Fig. 16a) and it is easily distinguished from the background, dominated by D-type asteroids. This is a quite robust family that remains isolated over a wide range of cutoff values, from 90 to $140 \mathrm{~m} \mathrm{~s}^{-1}$. Its distribution in the space of proper elements for $d_{\text {cut }}=130 \mathrm{~m} \mathrm{~s}^{-1}$ is shown in Fig. 15. It is worth noting that F07 studied this family and found that it is a D-type family. However, due to an incorrect choice of the cutoff level, all the 8 asteroids that they used to perform their classification are not actual members of the Panthoos family but of the Sergestus family. These two families merge together at $d_{\text {cut }}>140 \mathrm{~m} \mathrm{~s}^{-1}$. 

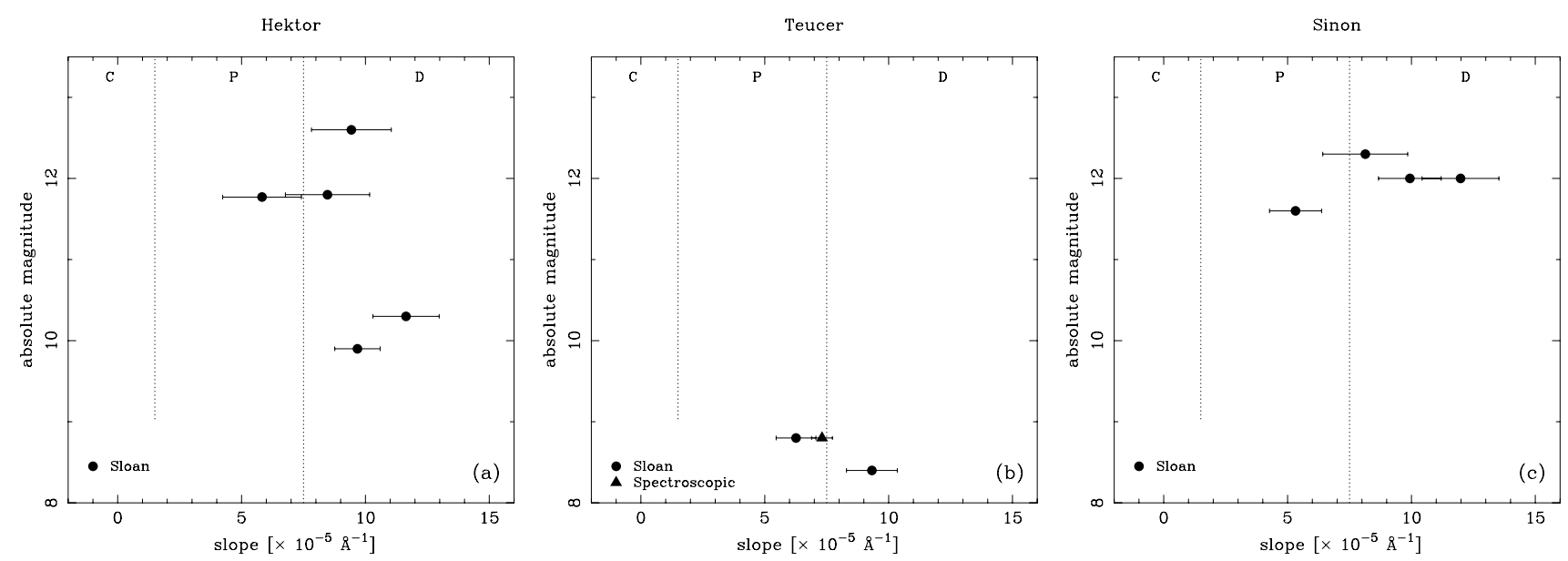

Fig. 14. Same as Fig. 12, but for three high-inclination families in the L4 swarm.

The Polydoros family is another example of a quite robust family, which is detected down to cutoff $90 \mathrm{~m} \mathrm{~s}^{-1}$. This family merges with the Sergestus family for $d_{\text {cut }} \geq 130 \mathrm{~m} \mathrm{~s}^{-1}$ to form the single Polydoros family shown in Fig. 15. The distribution of spectral slopes of the Polydoros + Sergestus family, shown in Fig. 16b, indicates that this is a quite homogeneous D-type family. Interestingly, the spectral slope of (4829) Sergestus measured by F07 indicates that this asteroid is likely to be a P-type, so it may be an interloper. Recall, however, that the Polydoros and Sergestus families are taxonomically indistinguishable from the background and this makes the discussion about interlopers difficult.

The Agelaos and 1999 RV165 families are somehow different from the other families of the Anchises clan. None of them survive down to small cutoffs and they appear less homogeneous in terms of taxonomy. Their distribution in proper elements is shown in Fig. 15. At $d_{\text {cut }}>135 \mathrm{~m} \mathrm{~s}^{-1}$, the Agelaos family incorporates asteroid (1173) Anchises and becomes the Anchises family. At the same cutoff, the 1999 RV165 family becomes the Antenor family after incorporating asteroid (2207) Antenor. These two families merge together at $d_{\text {cut }}>145 \mathrm{~m} \mathrm{~s}^{-1}$.

The 1999 RV165 family has only one member in the Sloan sample classified as P-type, so we cannot say too much about it. The Agelaos family has two members observed in the Sloan sample, one P- and one D-type, but this family has also been observed by F07 who identified it as the Anchises family. The distribution of spectral slopes of the Agelaos + Anchises family is shown in Fig. 16c. The largest member in this plot is (1173) Anchises $(H \simeq 8.9)$, and there is a correlation between spectral slope and size, already reported by F07, indicating that the larger the bodies the bluer the spectra. For the time being, we cannot say whether this correlation is real or just an artifact caused by a wrong definition of the family.

\subsubsection{Other families}

Figure 17 shows the distributions of spectral slopes in terms of absolute magnitude of five L5 families: Aneas, 1988 RG10, Asios, Phereclos, and Misenus.

The Aneas family has been studied by (Fornasier et al. 2004, hereafter F04) and also by F07, who treated it as the Sarpedon family. This family is actually formed from the merging of two families: Sarpedon and 1988 RN10. The Sarpedon family is resolved at $d_{\text {cut }}<130 \mathrm{~m} \mathrm{~s}^{-1}$, and the $1988 \mathrm{RN} 10$ family is resolved at $d_{\text {cut }}<140 \mathrm{~m} \mathrm{~s}^{-1}$. Both families are identified down

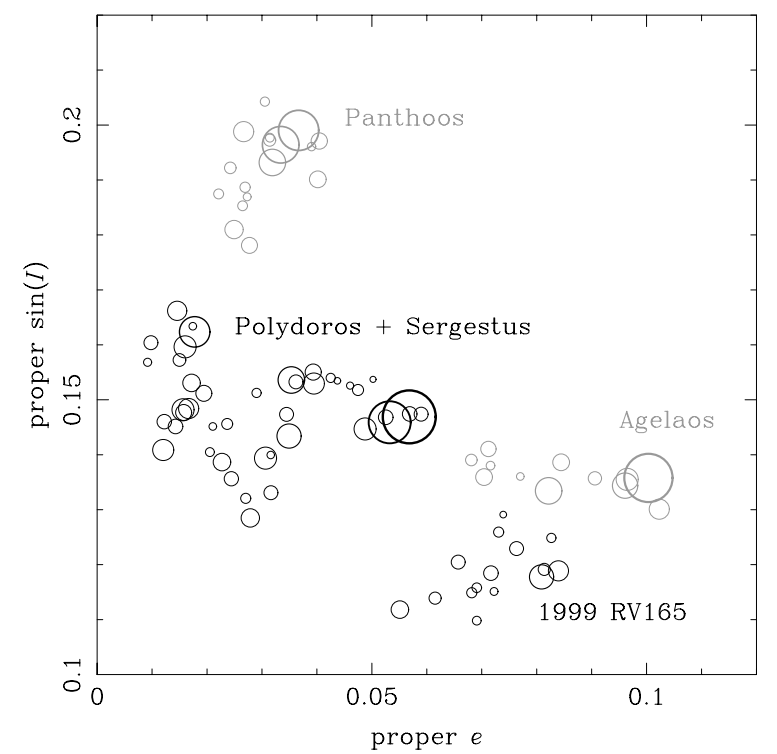

Fig. 15. Distribution in the space of proper elements of the Anchises clan as detected at $d_{\text {cut }}=130 \mathrm{~m} \mathrm{~s}^{-1}$. It is constituted by five families: Panthoos, Polydoros, Sergestus, Agelaos and 1999 RV165. (1173) Anchises is incorporated into the Agelaos family at $d_{\text {cut }}>135 \mathrm{~m} \mathrm{~s}^{-1}$. The size of each circle is proportional to the asteroid size.

to $d_{\text {cut }}=90 \mathrm{~m} \mathrm{~s}^{-1}$. In Fig. 17a we show the spectral slopes of the whole Aneas family (i.e., Aneas + Sarpedon + 1988 RN10). The values indicate that this is a quite homogeneous D-type family. The only two P-type members shown in Fig. 17a abandon the family at $d_{\text {cut }}<115 \mathrm{~m} \mathrm{~s}^{-1}$, so they are probably interlopers. So far, this family is one of the most homogeneous families in terms of taxonomy already detected, along with the Eurybates family in L4.

The 1988 RG10 and the Asios families, shown in Figs. 17b, c, have not been observed by spectroscopic surveys before. The distribution of Sloan slopes indicates that the 1988 RG10 family would be a quite homogeneous D-type family. For the Asios family the results are inconclusive. The Phereclos family, shown in Fig. 17d, has been analyzed by F04 and F07. The results point to a quite homogeneous D-type family. The only observation contained in the Sloan sample corresponds to asteroid (18940), already observed by F04, and its Sloan slope is compatible with its Spectroscopic slope. Finally, the Misenus family shown in Fig. 17e has no observations in the Sloan sample, but 

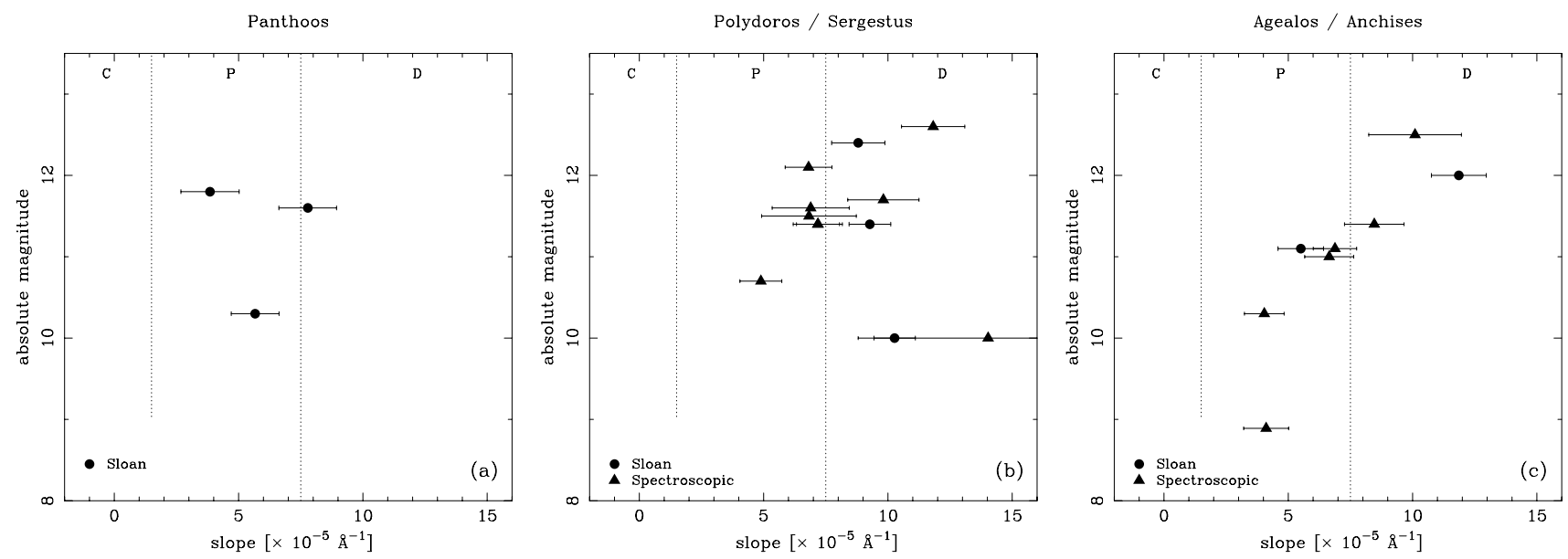

Fig. 16. Distribution of spectral slopes against absolute magnitude for three families of the Anchises clan: a) Panthoos, b) Polydoros + Sergestus, and c) Agelaos + Anchises. Full circles correspond to Sloan slopes. Triangles correspond to Spectroscopic slopes. The vertical dotted lines define the slope transition, within $\pm 0.8 \times 10^{-5} \AA^{-1}$, between the different taxonomic classes indicated above the plots.
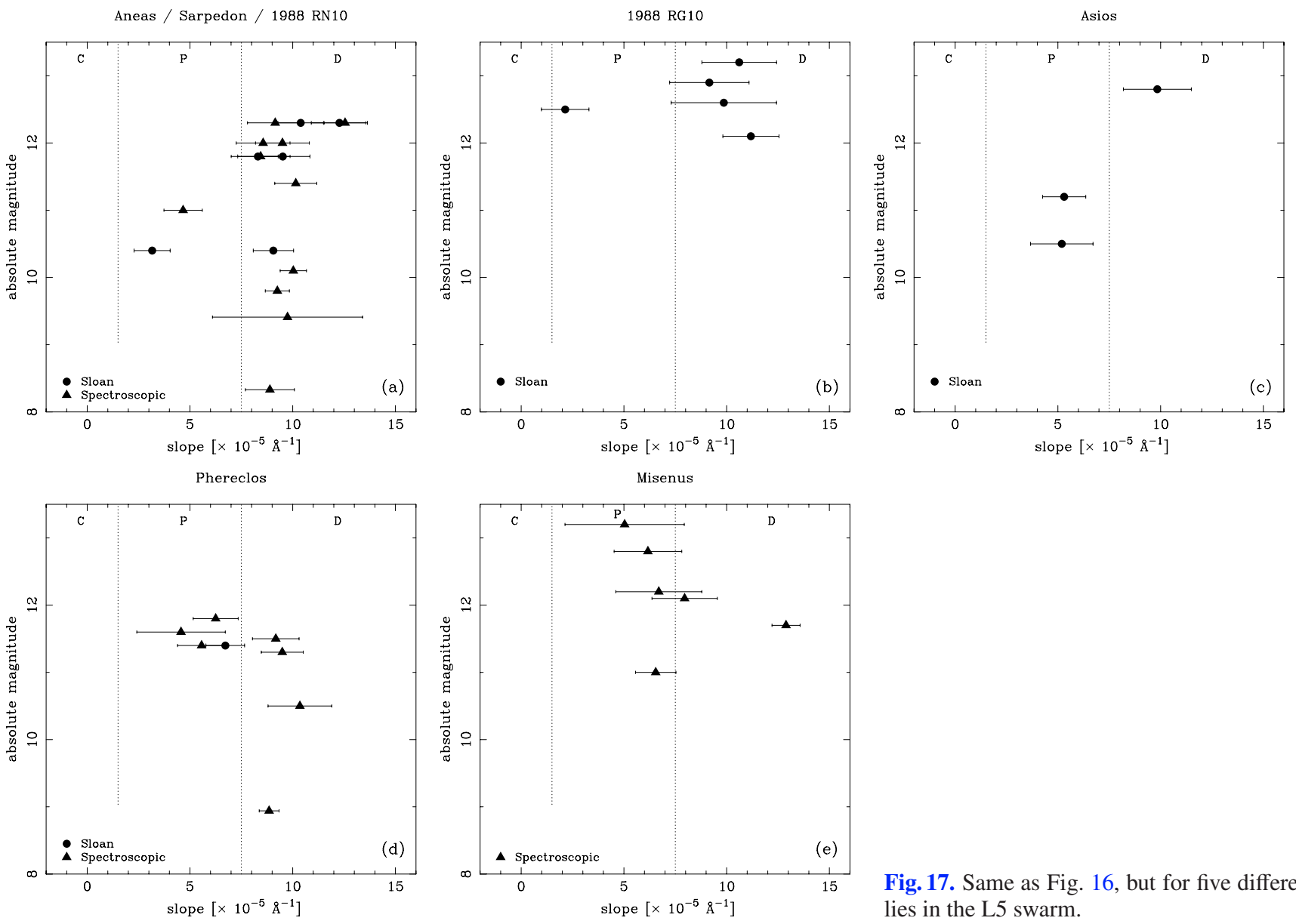

Fig. 17. Same as Fig. 16, but for five different families in the L5 swarm.

we include it here for the sake of completeness. It appears to be a P-type family.

It is worth noting that, as in the case of the L4 families, no systematic trend between spectral slope and size is appreciable among the individual L5 families.

\subsection{Discussion}

While the individual families in L5 appear to be taxonomically homogeneous, the individual families in L4 show a wide range of spectral slopes and a mixture of the C-, P-, and D-types. There are, at least, two possibilities for explaining the presence of different taxonomic classes within a single family:

- The family contains several interlopers, i.e., background asteroids that overlap with the family in proper elements. The amount of interlopers is significant because the presentlyobserved family members are so sparse that we need to use large cutoff values to detect the family. Therefore, only the families detected at small cutoffs, like the Eurybates family, can be considered, for the time being, as less contaminated 
by interlopers. Actually, we see that the Eurybates family would be the most homogeneous family of the Menelaus clan in terms of taxonomy. Note that the contamination by background interlopers would not introduce significant inhomogeneities in the taxonomy of L5 families, even if these families were not well defined, because most of these families are taxonomically indistinguishable from the background (in other words, the L5 families are dominated by D-type asteroids as well as the L5 background).

- We may invoke an aging process on the surfaces of the asteroids, such as space weathering, which causes the wide range of slopes observed within a single family. In this case, we must presume that the surfaces of the members of the family do not have the same age. In fact, many small members in the family may have been formed by secondary collisions, thus showing younger surfaces. Also, the small members, having a larger collision probability, may be more frequently affected by collisional resurfacing processes. Unfortunately, the space weathering is not well constrained in the case of the Trojan asteroids, and we cannot say whether it produces a reddening of the spectra with age, or viceversa (Moroz et al. 2004), or both. The lack of a clear correlation between spectral slope and size among the Trojan families, together with the still small amount of asteroids with known spectral slope, and the poorly-constrained collisional evolution of Trojan families, prevents us from performing a reliable analysis of any aging process. Moreover, the apparent taxonomical homogeneity of the L5 families seems to play against the surface aging scenario.

Nevertheless, the case of the Eurybates/Menelaus families constitutes an interesting paradigm of the possible effect of space weathering on the surfaces of Trojan asteroids. The compactness of the Eurybates family, compared to the Menelaus family, may be interpreted as a rough measure of youthfulness. There are many examples in the asteroid main belt that support this idea: the Karin family inside the Koronis family (Nesvorný et al. 2002), the Baptistina family inside the Flora clan (Bottke et al. 2007), and the Veritas family (Nesvorný et al. 2003). Within this hypothesis, the color distribution of the Eurybates/Menelaus families may be explained if we assume that space weathering causes a reddening of the surfaces with age. Then, we may speculate that the members of the Eurybates family are the fresh fragments from the interior of a former member of the Menelaus family. The remaining members of the Menelaus family would have much older surfaces thus being much redder. An analysis of the family ages based on purely dynamical/collisional arguments is mandatory to better address this issue.

\section{Conclusions}

We have analyzed the distribution of spectral slopes and colors of Trojan asteroids using a sample of data from the SDSS-MOC3 together with a collection of spectra obtained from several surveys. Our analysis has been focused on the Trojan asteroid families. We have studied the global properties of the sample as well as the properties of some individual families. Our results can be summarized as follows:

- The analysis of photometric data from the SDSS-MOC3 produces reliable results that are comparable with those obtained from the analysis of spectroscopic data.

- The distribution of spectral slopes among the Trojan asteroids shows a clear bimodality. About $2 / 3$ of the Trojan population is constituted by reddish objects that may be classified as D-type asteroids. The remaining bodies show less reddish colors compatible with the P-type, and only a small fraction (less than 10\%) is constituted of bodies with neutral colors compatible with the C-type.

- The members of asteroid families show a bimodal distribution with a very slight predominance of D-type asteroids. The background, on the contrary, is significantly dominated by D-type asteroids.

- The L4 and L5 swarms show significantly different distributions of spectral slopes. The distribution in L4 is bimodal with a slight predominance of D-type asteroids. The distribution in L5 is unimodal with a clear peak of D-type asteroids. These differences can be attributed to the presence of asteroid families.

- The background asteroids show the same spectral slope distributions in both swarms, with a significant fraction $(\sim 80 \%)$ of D-type asteroids. The families in L4 are dominated by Pand C-type asteroids, while the families in L5 are dominated by D-type asteroids.

- The background asteroids show correlations between spectral slope and orbital inclination and between spectral slope and size. D-type asteroids dominate among the highinclination bodies and also among the large bodies. Lowinclination bodies are slightly dominated by P-type asteroids. These correlations are most probably the result of the background collisional evolution, either by fragmentation or by collisional resurfacing. Similar correlations are not observed among the family members.

- We have also found evidence of a correlation between orbital inclination and size among the background asteroids, such that the largest bodies tend to be located at the highest inclinations.

- Individual families in the L5 swarm are taxonomically homogeneous, but in the L4 swarm show a mixture of taxonomic types. This may be attributed to the presence of interlopers or to a surface aging effect.

- Any taxonomic analysis of individual families must be accompanied by a detailed analysis of the families structure as a function of the cutoff level of detection. An estimation of the family ages is also mandatory to complement these analyses.

Acknowledgements. We wish to thank Sonia Fornasier, Elisabetta Dotto, Phillipe Bendjoya, and Alberto Cellino who kindly allowed us to use their spectroscopic data. We also wish to thank the referee, S. Fornasier, for her comments and suggestions. Fruitful discussions with Jorge Carvano are also highly appreciated. This work has been supported by CNPq (Brazil) and SECYT (Argentina).

\section{References}

Beaugé, C., \& Roig, F. 2001, Icarus, 153, 391

Bendjoya, P., Cellino, A., di Martino, M., \& Saba, L. 2004, Icarus, 168, 374 Binzel, R. P., Masi, G., \& Foglia, S. 2006, BAAS, 38, 627

Binzel, R. P., Masi, G., Foglia, S., et al. 2007, in Lunar and Planetary Institute Conference Abstracts, 38, 1851

Bottke, W., Vokrouhlicky, D., \& Nesvorny, D. 2007, in AAS/Division for Planetary Sciences Meeting Abstracts, 39, AAS/Division for Planetary Sciences Meeting Abstracts, \#50.02

Bus, S. J., \& Binzel, R. P. 2002, Icarus, 158, 146

Carvano, J. M., Mothé-Diniz, T., \& Lazzaro, D. 2003, Icarus, 161, 356

Dahlgren, M., Lagerkvist, C.-I., Fitzsimmons, A., Williams, I. P., \& Gordon, M. 1997, A\&A, 323, 606

Dotto, E., Fornasier, S., Barucci, M. A., et al. 2006, Icarus, 183, 420

Duffard, R., \& Roig, F. 2007, [arXiv: 0704. 0230]

Emery, J. P., \& Brown, R. H. 2003, Icarus, 164, 104

Fitzsimmons, A., Dahlgren, M., Lagerkvist, C. I., Magnusson, P., \& Williams, I. P. 1994, A\&A, 282, 634

Fornasier, S., Dotto, E., Marzari, F., et al. 2004, Icarus, 172, 221 
Fornasier, S., Dotto, E., Hainaut, O., et al. 2007, Icarus, 190, 622 Fukugita, M., Ichikawa, T., Gunn, J. E., et al. 1996, AJ, 111, 1748 Gil-Hutton, R., \& Brunini, A. 2008, Icarus, 193, 567

Ivezić, Ž., Tabachnik, S., Rafikov, R., et al. 2001, AJ, 122, 2749 Jewitt, D. C., \& Luu, J. X. 1990, AJ, 100, 933

Lazzaro, D., Angeli, C. A., Carvano, J. M., et al. 2004, Icarus, 172, 179

Luu, J., Jewitt, D., \& Cloutis, E. 1994, Icarus, 109, 133

Marzari, F., \& Scholl, H. 1998, A\&A, 339, 278

Marzari, F., Scholl, H., \& Farinella, P. 1996, Icarus, 119, 192

Marzari, F., Farinella, P., Davis, D. R., Scholl, H., \& Campo Bagatin, A. 1997, Icarus, 125,39

Marzari, F., Tricarico, P., \& Scholl, H. 2003, MNRAS, 345, 1091

Milani, A. 1993, Cel. Mech. Dyn. Astr., 57, 59

Morbidelli, A., Levison, H. F., Tsiganis, K., \& Gomes, R. 2005, Nature, 435, 462

Moroz, M., Baratta, G., Strazzulla, G., et al. 2004, Icarus, 170, 214
Nesvorný, D., Bottke, Jr., W. F., Dones, L., \& Levison, H. F. 2002, Nature, 417, 720

Nesvorný, D., Bottke, W. F., Levison, H. F., \& Dones, L. 2003, ApJ, 591, 486

Roig, F., \& Gil-Hutton, R. 2006, Icarus, 183, 411

Roig, F., Nesvorny, D., Gil-Hutton, R., \& Lazzaro, D. 2008, Icarus, 194, 125

Schwarz, R., Gyergyovits, M., \& Dvorak, R. 2004, Cel. Mech. Dyn. Astr., 90, 139

Szabó, G. M., Ivezić, Ž., Jurić, M., \& Lupton, R. 2007, MNRAS, 377, 1393

Tholen, D. J. 1989, in Asteroids II, ed. R. P. Binzel, T. Gehrels, \& M. S. Matthews (Tucson, AZ: Univ. Arizona Press), 1139

Xu, S., Binzel, R. P., Burbine, T. H., \& Bus, S. J. 1995, Icarus, 115, 1

Yang, B., \& Jewitt, D. 2007, AJ, 134, 223

Zappalà, V., Bendjoya, P., Cellino, A., Farinella, P., \& Froeschlé, C. 1995, Icarus, 116,29

Zellner, B., Tholen, D. J., \& Tedesco, E. F. 1985, Icarus, 61, 355 